\title{
Shape-Persistent Phenylene-Ethynylene Macrocycles Spectroscopy 2 and Dynamics: From Molecules to the Hydrogen-Bonded Organic ${ }_{3}$ Framework Material
}

${ }_{4}$ Eduardo Gomez, Maria Rosaria di Nunzio, Miquel Moreno, Ichiro Hisaki, ${ }^{*}$ and Abderrazzak Douhal*

Cite This: https://dx.doi.org/10.1021/acs.jpcc.0c01369

Read Online

5 ABSTRACT: Shape-persistent phenylene-ethynylene macrocycles 6 are being intensively studied since they are being used for making 7 hydrogen-bonded organic frameworks (HOFs). Here, we report 8 on studies of two expanded macrocycles, $\mathrm{Ex}-\mathrm{COOH}$ and Ex9 COOMe (its methyl ester), in solutions and in the solid state 10 (HOF Ex-apo and Ex-ester, respectively). For Ex-COOH, the 11 theoretical results suggest the occurrence of an excited-state 12 intramolecular charge-transfer in agreement with the experimental 13 observation. While this event occurs in $<200 \mathrm{fs}$ in $\mathrm{Ex}-\mathrm{COOH}$ in 14 DMF solution, it becomes longer ( 2.5 ps) in Ex-COOMe. The 15 formed species in the latter case shows a fluorescence lifetime of $162.02 \mathrm{~ns}$, however for Ex-COOH, we found lifetimes of 0.4, 2.2, and

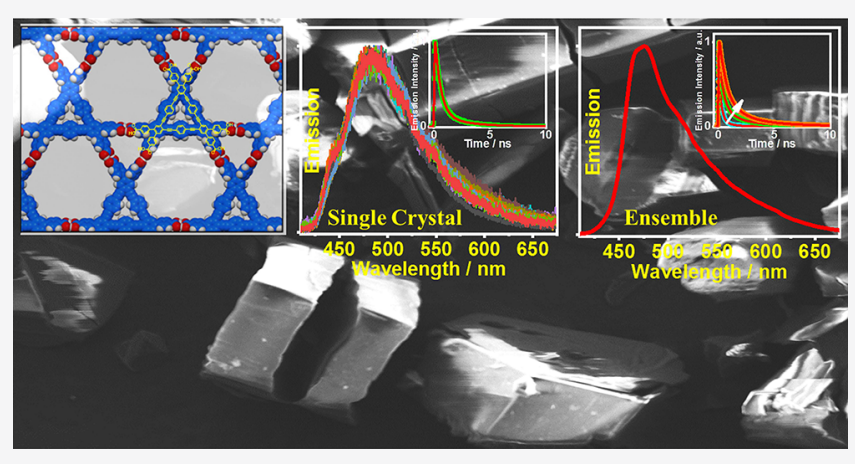
175.95 ns. Nanosecond-flash photolysis studies of Ex-COOH reveal two triplets having lifetimes of 0.45 and $2 \mu$ s. The ensemble solid 18 studies indicate that their photobehaviors are affected by the presence of different fluorescent emitters. Moreover, at the single 19 crystal level, the emission spectra and decays of washed HOF Ex-apo crystals do not depend on the interrogated site. These results 20 provide new insights into the photobehavior of both macrocyclic units of HOF Ex-apo. The findings will help for future researches 21 and development of smart devices based on this kind of materials.

\section{INTRODUCTION}

22 Porous molecular crystals constructed through intermolecular 23 H-bonds are often called hydrogen-bonded organic frame24 works (HOFs). ${ }^{1-6}$ During the past decade, these new materials 25 have attracted the interest of the scientific community due to 26 their exceptional properties and applications. ${ }^{7-10}$ Unlike 27 metal-organic frameworks (MOFs) ${ }^{11-15}$ and covalent28 organic frameworks (COFs), ${ }^{16-20}$ which still remain the 29 most studied materials due to their potential application in 30 different fields of science and technology, ${ }^{21-24}$ HOFs are 31 constructed only by organic molecules through non-covalent 32 H-bonding interactions. ${ }^{25-28}$ As any other porous material, 33 both the pore sizes and structures are conditioned by the used 34 linkers or molecular units, ${ }^{9}$ giving the formed network different 35 properties and applicability life in gas storage and separation, 36 explosive detection, and optoelectronics, to cite a few of 37 them. $^{25,29-32}$ For example, the luminescent HOF-1111 38 (composed by a tetraphenylethylene derivative molecular 39 unit) is able to detect several aromatic compounds, including 40 nitroaromatic explosives. ${ }^{25}$ Recently, we have reported on the 41 dynamics and spectroscopic studies of two HOFs (CBPHAT42 la and CPHATN-1a), constructed by hexaazatriphenylene 43 derivatives. ${ }^{33,34}$ We showed the occurrence of both proton44 and charge-transfer reactions and a remarkable response to $45 \mathrm{HCl}$ vapors. ${ }^{34}$ Another $\mathrm{HOF}$, based on $4,4^{\prime}, 4^{\prime \prime}, 4^{\prime \prime \prime}$-tetra $(2,4-$ diamino-1,3,5-triazin-6-yl)tetraphenylethene (HOF-5), has 46 shown a certain character of flexibility, making it a potential 47 candidate for gas adsorption and separation. ${ }^{35}$ HOFs of large 48 pores or channels capable of encapsulating large molecules, like 49 drugs are being exploited by the scientific community. ${ }^{36,37}$ For 50 example, PFC-1 HOF, can efficiently encapsulate doxorubicin 51 (Doxo) for synergistic chemophotodynamic therapy, showing 52 high therapeutic efficiency and lower cytotoxicity. ${ }^{36}$ Hence, 53 studying new HOFs with large pore sizes is paramount for 54 their potential use in diverse applications in science and 55 technology.

A few years ago, a new kind of HOFs, based on 57 dehydrobenzoannulenes (DBAs) displaying high planarity 58 and large $\pi$-conjugation, have emerged as possible candidates 59 for photonic applications. ${ }^{38-40}$ For example, HOF T12-apo 60 displays a long fluorescence lifetime $(25 \mathrm{~ns})$ and interesting 61 CIE coordinates $(0.42,0.55)$, which make it a potential 62 candidate to fabricate white light-emitting diode devices. ${ }^{32}$ The 63

Received: February 17, 2020

Revised: February 28, 2020

Published: March 2, 2020 

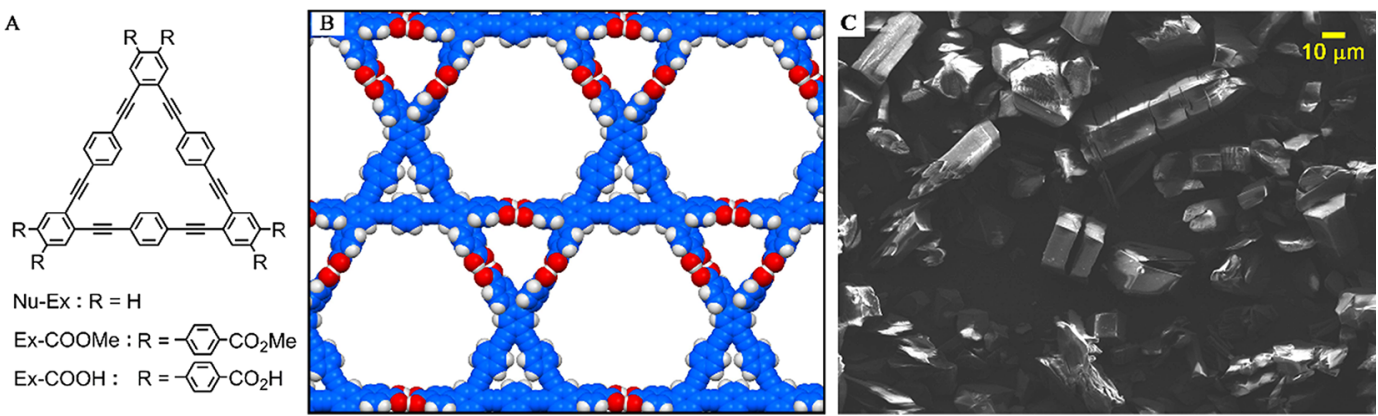

Figure 1. (A) Molecular structures of Nu-Ex, Ex-COOMe, and Ex-COOH. (B) Hydrogen-bonded network of Ex-apo. (C) SEM image of the HOF Ex-apo formed by Ex-COOH.

64 judicious design of the molecular structure of these DBAs is 65 paramount for the control of the crystallinity and pore size of 66 the HOF governed by $\mathrm{H}$-bonds and $\pi-\pi$ interactions between 67 the molecular units. ${ }^{41}$ To this end, one can change the pore 68 size and the electronic structure of the core of the fundamental 69 units. Indeed, most of the research efforts have been focused 70 on the synthesis of new HOFs and on the control of their 71 porous structure. ${ }^{9,27,30,42,43}$ On the other hand, the scientific 72 literature is still missing a more comprehensive advance in their 73 photodynamics. Time-resolved (femtosecond to nanoseconds 74 regime) studies of the photoprocesses occurring in HOFs will 75 provide concise information on the photoevents that shape 76 their photobehavior, as it has been done, for example, in silica77 based materials and their composites. ${ }^{44}$ In addition to this, 78 further research in this direction will improve the knowledge in 79 the related fields in science and technology. For example, 80 investigating the photophysics and photochemistry of HOFs 81 may lead to the development of HOFs-based optoelectronic 82 devices, sensors, and drug delivery. ${ }^{45-47}$

83 Here, we report on experimental and theoretical studies of 84 two new $\pi$-conjugated systems, more expanded than DBAs, 85 namely, phenylene-ethynylene macrocycles, $\mathrm{Ex}-\mathrm{COOH}$ and its 86 methyl ester derivative (Ex-COOMe), in N,N-dimethylforma87 mide (DMF) solutions and in the solid state. While the UV88 visible absorption and emission spectra of Ex-COOH depend 89 on its concentration, the ones of Ex-COOMe are independent. 90 This suggests the existence of different $\mathrm{Ex}-\mathrm{COOH}$ species at 91 the ground state $\left(S_{0}\right)$, which are generated by the interactions 92 between its carboxylic acid groups and the DMF molecules. 93 For both molecules in DMF, an intramolecular charge-transfer 94 (ICT) reaction occurs at the $S_{1}$ state, which is fast $(2.5 \mathrm{ps})$ for $95 \mathrm{Ex}-\mathrm{COOMe}$ and ultrafast $(<200 \mathrm{fs})$ for Ex-COOH. The 96 theoretical results support the H-bond interactions of Ex$97 \mathrm{COOH}$ with DMF molecules and the occurrence of an ICT 98 process at the $S_{1}$ state. The ICT species of the methyl ester 99 derivative have a fluorescence lifetime of $2.02 \mathrm{~ns}$, while those of $100 \mathrm{Ex}-\mathrm{COOH}$ show lifetimes of 400 ps, $2.23 \mathrm{~ns}$, and $5.95 \mathrm{~ns}$. The $101 \mathrm{~ns}-\mu \mathrm{s}$ flash photolysis experiments on $\mathrm{Ex}-\mathrm{COOH}$ in DMF 102 show two triplet lifetimes of 0.45 and $2 \mu$ s. In the solid state, 103 Ex-ester and HOF Ex-apo exhibit ICT reactions producing 104 emitters of different fluorescence lifetimes. HOF Ex-apo 105 exhibits an additional fluorescence lifetime of $4.8 \mathrm{~ns}$ as a 106 result of $\mathrm{H}$-bond interactions between the $\mathrm{Ex}-\mathrm{COOH}$ units 107 forming the crystal structure. Moreover, fluorescence micros108 copy experiments on washed single crystals indicate that the 109 photobehavior does not depend on the interrogated crystal 110 site. These results provide new findings for a better 111 understanding of the photobehavior of both phenylene- ethynylene macrocycle-based molecules and related HOFs, 112 and this knowledge may help in the design of new HOFs for 113 potential applications in photonics or drug photodelivery. Our 114 previous reports as well as this one are contributing to the 115 understanding of the spectroscopic properties of these novel 116 HOF materials. ${ }^{32-34,47,48}$

\section{EXPERIMENTAL SECTION}

Synthesis and characterization of the molecular building blocks 118 units, Ex-COOMe and Ex-COOH (Figure 1A), and the $119 \mathrm{fl}$ constructed materials (Ex-ester and HOF Ex-apo) were 120 previously reported. $^{49}$ The anhydrous solvent N,N-dimethyl- 121 formamide (99.8\% spectroscopic grade, DMF) was purchased 122 from Sigma-Aldrich and used as received.

123

The theoretical calculations have been carried out at the 124 DFT level for the ground $\left(\mathrm{S}_{0}\right)$ and lowest triplet $\left(\mathrm{T}_{1}\right)$ states, 125 whereas the time-dependent DFT (TDDFT) formalism was 126 used to optimize and analyze the lowest singlet excited 127 electronic state $\left(S_{1}\right)$. All the calculations have been performed 128 using the long-range corrected hybrid functional CAM- 129 $\mathrm{B}^{2} \mathrm{LYP}^{50}$ that minimizes the error of internal CT excitations 130 that would appear at an unphysical too low energy. The basis 131 set used is the split-valence 6-31G(d,p), which adds up 132 polarization ( $\mathrm{d}$ and $\mathrm{p}$ functions) for all the atoms. ${ }^{51,52}$ For Ex- 133 $\mathrm{Nu}$, we have verified that a further increase in the basis set (6- 134 $311+G(d, p))$ does not significantly modify the results for $S_{0} 135$ and $T_{1}$ states. The introduction of diffuse $(+)$ functions leads 136 to the appearance of some lowest electronic excited states with 137 unphysical Rydberg character. Full optimization of the 138 different states has been performed without any symmetry 139 restriction. The solvent (DMF) effect has been introduced 140 through the self-consistent PCM continuum method that 141 defines a cavity of solvent (with a specific dielectric constant) 142 where the molecule (solute) is introduced. The polarization of 143 the molecule by the surrounding DMF solvent is considered in 144 the self-consistent procedure. ${ }^{5,54}$ All the calculations have 145 been carried out using the Gaussian09 suite of programs. ${ }^{55} 146$ This same methodology was previously proved valid for the 147 analysis of a molecular system similar to the one studied 148 here. ${ }^{56}$ In that work, we also compared the results obtained 149 with CAM-B3LYP against more conventional functionals such 150 as B3LYP or PBE0 by performing calculations on the related 151 $\mathrm{Nu}-\mathrm{T} 12$ core model. Very similar results were found in the 152 calculations of the lowest excited states. Thus, the problem of 153 unphysical low energy states appears not to be relevant for this 154 family of compounds. For the small Nu-T12 model, we also 155 verified that a larger basis set $(6-311 G(d, p))$ does not 156 appreciably modify the energies of the analyzed electronic 157 
158 states, and we believe that the methodology used here leads to 159 an accurate picture of the energy diagram of this family of 160 compounds.

161 Steady-state UV-visible absorption and emission spectra 162 have been recorded using JASCO V-670 and FluoroMax-4 163 (Jobin-Yvon) spectrophotometers, respectively. For diluted, 164 intermediate, and concentrated samples of $\mathrm{Ex}-\mathrm{COOH}$ in DMF 165 solutions, the optical densities (ODs) at $355 \mathrm{~nm}$ in a $1 \mathrm{~cm}$ cell 166 were $0.05,0.35$, and 1.78 , respectively.

167 Picosecond (ps) emission decays were measured using a 168 time-correlated single photon counting (TCSPC) system. ${ }^{57}$ 169 The samples were excited at $371 \mathrm{~nm}$ by a $40 \mathrm{ps}$ pulsed diode 170 laser $(<5 \mathrm{~mW}, 40 \mathrm{MHz}$ repetition rate), and the instrument 171 response function (IRF) was $\sim 70$ ps. The experimental data 172 were analyzed using the FLUOFIT package (PicoQuant), and 173 the fit procedure is described elsewhere. ${ }^{5}$

174 The nanosecond (ns) flash photolysis setup has been 175 described previously. ${ }^{58}$ Briefly, it consists of a LKS.60 laser 176 flash photolysis spectrometer (Applied Photophysics) and a 177 Vibrant (HE) 355 II laser (Oppotek). To excite the sample, we 178 used the third harmonic output $(355 \mathrm{~nm})$. The pump fluence 179 was $\sim 80 \mathrm{~mJ} \mathrm{~cm}^{-2}$. As a probe light, we used the output for a $180150 \mathrm{~W}$ Xenon arc lamp. The light transmitted through the 181 sample was then dispersed by a monochromator and detected 182 by a visible photomultiplier (Applied Photophysics R928), 183 coupled to a digital oscilloscope (Agilent Infiniium DS08064A, $184600 \mathrm{MHz}, 4 \mathrm{GSa}$ s1). The measured IRF of the system is $\sim 8$ $185 \mathrm{~ns}$. All the experiments were performed at $293 \mathrm{~K}$ and in a $1 \mathrm{~cm}$ 186 cell.

187 The femtosecond (fs) emission transients have been 188 collected using the fluorescence up-conversion technique. ${ }^{47}$ 189 The system consists of a fs Ti:Sapphire oscillator MaiTai HP 190 (Spectra Physics) coupled to a second harmonic generator and 191 an up-conversion setup. ${ }^{59}$ The oscillator pulses (90 fs, 250 $192 \mathrm{~mW}, 80 \mathrm{MHz}$ ) are centered at $740 \mathrm{~nm}$ and doubled in an 193 optical setup through a $0.5 \mathrm{~mm} \mathrm{BBO}$ crystal to generate a 194 pumping beam at $370 \mathrm{~nm}(\sim 0.1 \mathrm{~nJ} /$ pulse $)$. The polarization of 195 the latter was set to magic angle with respect to the 196 fundamental beam. The IRF of the setup is $200 \mathrm{fs}$, and with 197 a deconvolution procedure, we can resolve components of $\sim 50$ 198 fs.

199 The fluorescence lifetime imaging (FLIM) measurements 200 were performed on a MicroTime 200 confocal microscope. ${ }^{32}$ 201 As an excitation source, we used a diode laser (40 ps) with an 202 excitation wavelength of $371 \mathrm{~nm}$. For the anisotropy 203 measurements, the light was passed through a polarizing 204 beam splitter that allows for a simultaneous detection of the 205 parallel and perpendicular signals emission. A G-factor of 1.2, 206 accounting for differences in the detection sensitivity for both 207 polarizations in the setup, was used in the calculation of the 208 anisotropy. The limits for the anisotropy range from -0.5 to 2091.0 , in which -0.5 corresponds to perpendicular orientation 210 and 1.0 to parallel orientation. The emission signal was 211 collected using a long-pass filter (HQ430LP Chroma).

\section{RESULTS AND DISCUSSION}

212 3.1. Theoretical Calculations. For a comprehensive 213 theoretical study of the $\mathrm{Ex}-\mathrm{COOH}$ molecule, we have also 214 performed the calculations for the pristine core $(\mathrm{Nu}-\mathrm{Ex}$; see 215 Figure 1A). The theoretical analysis has been done in the gas 216 phase and taking into account the solvent (DMF) effect.

217 To begin with, the full optimization of the $\mathrm{Nu}$-Ex system 218 inside a continuum cavity of DMF has been carried out at $S_{0}$,
$\mathrm{S}_{1}$, and $\mathrm{T}_{1}$ states. Table $\mathrm{S} 1$ shows the atomic distances. 219 Practically, these distances do not change from a state to 220 another one, indicating that the geometry of the molecule is 221 practically the same in these electronic states. As we can see in 222 Figure $2 \mathrm{~A}$, the geometry of $\mathrm{Nu}-\mathrm{Ex}$ is not fully planar since the $223 \mathrm{f} 2$

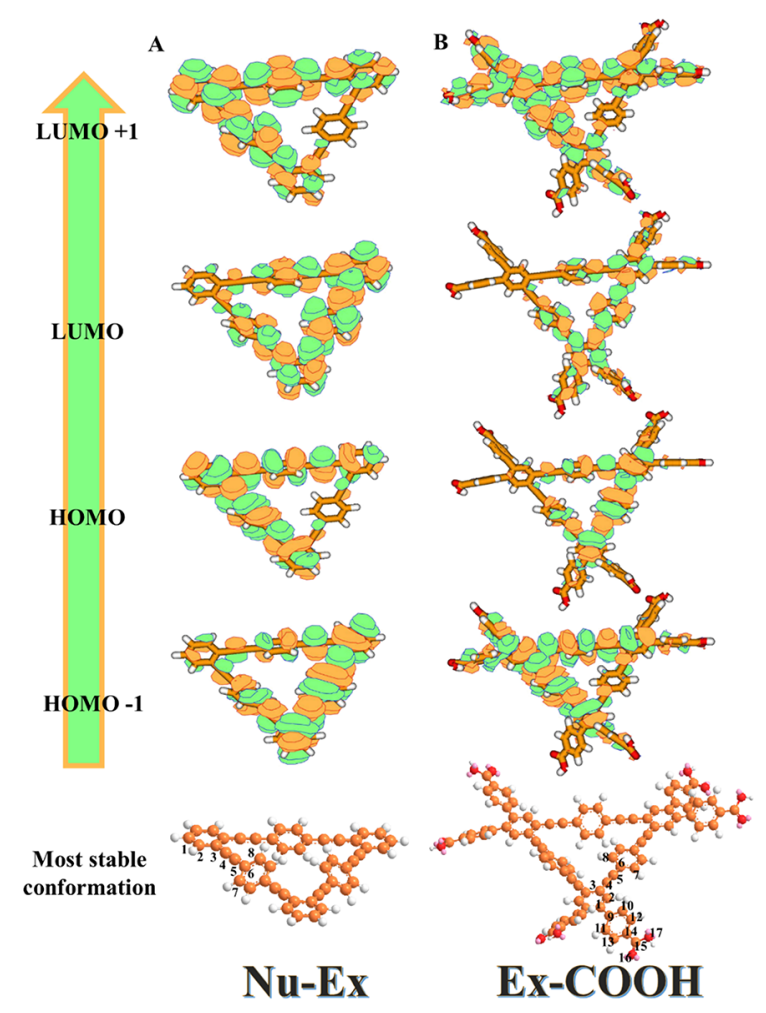

Figure 2. Frontier molecular orbitals involved in the electronic states of (A) Nu-Ex and (B) Ex-COOH molecules. The molecular structures and the numeration of the atoms used in Tables S1-S4 are shown at the bottom of the figure.

central phenyl groups are slightly out-of-plane, so the molecule 224 does not possess any symmetry. While one of the phenyls is 225 almost in the global plane of the molecule, two of them are 226 about $21^{\circ}$ out-of-plane. In this case, the dipole moment is 227 almost null because the phenyl groups have no dipole moment. 228 The loss of symmetry is reflected in the shape of the frontier 229 molecular orbitals: they spread over the full molecule but with 230 different magnitudes along the three sides of the triangle 231 (Figure 2A). The $\mathrm{S}_{0}$ and $\mathrm{T}_{1}$ states reflect two main electronic 232 excitations involving the HOMO-1, HOMO, LUMO, and 233 LUMO +1 orbitals. The results also show that all the orbitals 234 implied in the lowest electronic excitations are $\pi$-type orbitals. 235 Therefore, the $S_{1}$ state has a $\pi-\pi^{*}$ character. Even if Nu-Ex is 236 not fully symmetric, it almost fits to the $D_{3 h}$ symmetry group, 237 and symmetry considerations lead to the conclusion that the $S_{0} 238$ $\rightarrow S_{1}$ transition has a very small oscillator strength. The lack of 239 a high degree of symmetry explains why the oscillator strength 240 $(f)$ is not exactly zero. Moreover, the $S_{2}$ state, which has a high 241 probability to be accessed from $S_{0}$, is almost degenerate with $S_{3} 242$ (within $D_{3 h}$ symmetry, $S_{2}$ would be doubly degenerate). It is 243 worth noting that excitation to $S_{2}$ (and $S_{3}$ ) involves the same 244 molecular orbitals as those of excitation to $S_{1}$. Upon geometry 245 optimization at $S_{1}$, there is an increase in the deviation from 246 the planar symmetry. So, $S_{1}$ now can be accessed from $S_{0}$ as the 247 oscillator strength is larger than 1 . Transitions to $S_{2}$ and the 248 
249 almost degenerate $S_{3}$ states still have a larger probability $(f>$ 2502 ). As expected, $T_{1}$ has the same $\pi-\pi^{*}$ nature as that of $S_{1}$. 251 Figure S1A shows a schematic representation of the energy 252 levels of the different electronic states of $\mathrm{Nu}-\mathrm{Ex}$ in DMF. We 253 found that the difference between the $S_{0}$ and $T_{1}$ states in the 254 gas phase is $61.96 \mathrm{kcal} / \mathrm{mol}(2.68 \mathrm{eV})$, while this value in DMF 255 decreases until $56.15 \mathrm{kcal} / \mathrm{mol}(2.43 \mathrm{eV})$. The energy gap $256(5.81 \mathrm{kcal} / \mathrm{mol})$ clearly diminishes upon the introduction of 257 DMF (continuum model), revealing the polarity effect of the 258 solvent molecules surrounding $\mathrm{Nu}-\mathrm{Ex}$.

259 Table S2 shows the values of Mulliken charges of the 260 corresponding carbon atoms including those of the atoms 261 attached to them, where we use the numeration shown in 262 Figure 2A. All the values are always quite small. Moreover, they 263 are also quite similar at the electronic states of the same 264 calculation: gas phase and DMF. We notice also small 265 differences when comparing the effect of the solvent: globally, 266 it increases the charge separation, as expected, but the 267 differences are insignificant. Comparing both triplet states, 268 the charges in the presence of DMF are slightly larger than 269 those in the gas phase. The charges at $S_{1}$ are closer to the ones 270 of the triplet, especially when the geometry of $S_{1}$ is optimized 271 (last column of Table S2). Interestingly, the value on carbon 272 atom 5 at $S_{0}$ in both calculations is relatively large and negative. 273 However, at $S_{1}$ and $T_{1}$, the negative charge of carbon atom 5 is 274 smaller, although it is now shared with carbon atom 6 , so the 275 sum of charges of carbons 5 and 6 is almost the same at the 276 electronic states. For carbon atom 3, there is noticeable a 277 change of the sign upon different electronic excitations (from 278 positive to negative). This change only takes place when the 279 geometry is optimized at the excited state. However, the 280 absolute values of the charges are quite small, so this change 281 may not have any relevance.

282 We have carried out a similar theoretical study for the larger 283 system, Ex-COOH. The optimized geometry at $\mathrm{S}_{0}$ shows that 284 the molecule is not planar like Nu-Ex (Figure 2B). Table S3 285 shows the distances between the atoms at $S_{0}, S_{1}$, and $T_{1}$. As we 286 have observed for $\mathrm{Nu}-\mathrm{Ex}$, the distances between atoms are not 287 significantly different, suggesting that geometry is not affected 288 by the electronic excitation. However, in this case, the 1,4289 phenylene rings are out-of-plane and not symmetrically 290 displaced from the planarity. This leads to an increase in the 291 asymmetry of the molecule in comparison with $\mathrm{Nu}-\mathrm{Ex}$. This 292 change is also reflected in the shape of the frontier molecular 293 orbitals (Figure 2B). Again, the vertical Franck-Condon 294 excitation shows a quite small oscillator strength of the $S_{0} \rightarrow S_{1}$ 295 transition $(f=0.07)$. As in the Nu-Ex model, the transition 296 mainly comes from two excitations that involve the HOMO-1, 297 HOMO, LUMO, and LUMO+1 orbitals (Figure 2B). These 298 orbitals are clearly identified as $\pi$-orbitals, and so, again, the 299 small transition probability has to be ascribed to the "almost $300 D_{3 h}$ " symmetry of the molecule. Like the $\mathrm{Nu}-\mathrm{Ex}$ case, the 301 optimization of Ex-COOH at the excited state leads to a less 302 symmetric geometry, and the final geometry shows a relatively 303 large $(f=1.59) S_{0} \rightarrow S_{1}$ transition, while the transition to $S_{2}$ 304 (and the almost degenerate $S_{3}$ ) maintains a clearly larger 305 probability $(f=2.92)$, even though the orbitals involved in the 306 excitations to $S_{2}$ and $S_{3}$ are the same as those involved in the $S_{0}$ $307 \rightarrow S_{1}$ transition. This observation will be later demonstrated 308 experimentally with the absorption spectrum, which shows the 309 allowed $S_{0} \rightarrow S_{1}$ transition. The shape of the molecular orbitals 310 involved in the excitation to $S_{1}$ leads to predict a quite modest 311 degree of CT upon electronic excitation. All the orbitals involved in the lowest electronic excitations studied here are 312 clearly identified as $\pi$-orbitals. Therefore, in the absence of 313 symmetry, these excitations are symmetry-allowed. The spread 314 of the $\pi$-orbitals shown in Figure 2B suggests a possible degree 315 of CT upon excitation, as we will see in the experimental part. 316

Another consequence of the extended asymmetry of Ex- 317 $\mathrm{COOH}$ is that, in this case, the dipole moment is not null, as 318 $\mathrm{Nu}$-Ex, being 7.16 and $7.05 \mathrm{D}$ in the gas phase at $\mathrm{S}_{0}$ and $\mathrm{T}_{1}, 319$ respectively. Curiously, both electronic states have almost 320 equal dipole moments, and this is the main reason why the 321 solvent does not modify the relative energy difference between 322 $\mathrm{S}_{0}$ and $\mathrm{T}_{1}$, being in both situations (gas phase and continuum 323 method DMF media) $\sim 55 \mathrm{kcal} / \mathrm{mol}$, contrary to what we 324 observed for the small $\mathrm{Nu}$ model. Figure $\mathrm{S} 1 \mathrm{~B}$ shows a 325 schematic representation of the energy levels of Ex-COOH 326 using the continuum method. As we can see, the energy 327 difference $(1.05 \mathrm{eV})$ between the $S_{1}$ and $T_{1}$ states is smaller 328 than the one for $\mathrm{Nu}-\mathrm{Ex}(1.49 \mathrm{eV})$. In DMF, the dipole 329 moments have values of $10.24 \mathrm{D}\left(\mathrm{S}_{0}\right), 10.27 \mathrm{D}\left(\mathrm{T}_{1}\right), 10.22 \mathrm{D} 330$ $\left(\mathrm{S}_{1(\mathrm{FC})}\right)$, and $10.14 \mathrm{D}\left(\mathrm{S}_{1}\right)$. Again, these values do not present 331 significant differences, confirming the previous observations. 332

As for the Mulliken charge analysis, now there are more 333 noticeable charge separations between different parts of the 334 molecule (Table S3, and the numeration of the atoms is shown 335 in Figure 2B). Not surprisingly, the carboxylate groups present 336 large charge separation between the carbon and oxygen atoms. 337 Again, the solvent effect is quite small, usually increasing a little 338 bit the charges but with some exceptions. The negative charge 339 value on carbon atom 5 is still quite high, and it even increases 340 a little bit as compared with the one obtained for Nu-Ex. The 341 $S_{1}$ and $T_{1}$ states show very similar charges, which are not very 342 much different from the ones of the ground state. This is 343 especially true for the charges on the branches. The charges on 344 the core are also similar and are not significantly different from 345 the ones obtained for $\mathrm{Nu}-\mathrm{Ex}$ that does not have the "benzoic" 346 branches. It can be then concluded that the enlargement of the 347 molecule does not significantly affect the electronic structure of 348 the core. This is in agreement with the quite similar energies of 349 the different states of both $\mathrm{Nu}-\mathrm{Ex}$ and $\mathrm{Ex}-\mathrm{COOH}$. However, 350 the global molecule is not planar, and the dipole moment 351 changes from almost 0 to $10 \mathrm{D}$.

An interesting point that has been also theoretically analyzed 353 is the possibility of a direct interaction (H-bond) of Ex-COOH 354 with DMF molecules. In this sense, we have calculated the 355 optimized structure with up to six DMF molecules, H-bonded 356 to Ex-COOH. The analysis of the interaction with only one 357 DMF molecule reveals that, as expected, the interaction implies 358 the acid hydrogen atom of the carboxylic acid of $\mathrm{Ex}-\mathrm{COOH} 359$ and the oxygen atom of DMF. When we added one molecule 360 of DMF, we observed a large stabilization of $16.57 \mathrm{kcal} / \mathrm{mol} 361$ (Table S5). By subsequent addition of DMF molecules, we still 362 increase the stabilization until all the acid groups of Ex-COOH 363 are each interacting with one DMF molecule. In this case, we 364 obtained a stabilization of $97.44 \mathrm{kcal} / \mathrm{mol}$. Scheme S1 shows 365 the optimized structure of Ex-COOH interacting with six 366 molecules of DMF. H-bonds between the solute and solvent 367 molecules and related proton-transfer reactions are largely 368 studied from the point of view of theory and experiment. ${ }^{60-62} 369$ The $\mathrm{O} \cdots \mathrm{H} \cdots \mathrm{O} \mathrm{H}$-bond is quite strong as the $\mathrm{O} \cdots \mathrm{H}$ distance is 370 1.60 A. Figure $S 1$ discloses an additional (weaker) interaction 371 of the hydrogen atom of the $\mathrm{CHO}$ group of DMF with the 372 other carboxylic oxygen of Ex-COOH. The $\mathrm{O} \cdots \mathrm{H}$ distances are 373 now quite longer $(2.24 \AA)$, so this additional interaction is 374 
375 quite weak (and not present for the full six DMF molecules as 376 shown in Scheme S1). In brief, the theoretical calculations of $377 \mathrm{Ex}-\mathrm{COOH}$ in DMF suggest that (i) the optimized geometry of 378 the molecule is not planar, making the $S_{0} \rightarrow S_{1}$ transition 379 possible. (ii) An ICT reaction occurs at $S_{1}$. (iii) In DMF, the 380 interactions between the acid groups of $\mathrm{Ex}-\mathrm{COOH}$ and the 381 solvent molecules stabilize the formed complexes, giving the 382 possibility to a co-existence of different structures. We will use 383 this knowledge for a better understanding of the experimental 384 findings.

\section{3.2. Steady-State Absorption and Emission Studies in}

386 Solution. To begin with, Figure 3 shows the UV-visible 387 absorption and emission spectra of Ex-COOMe and Ex$388 \mathrm{COOH}$ in DMF solutions at different concentrations (ODs at $389355 \mathrm{~nm}$ are $0.05,0.35$, and 1.78).

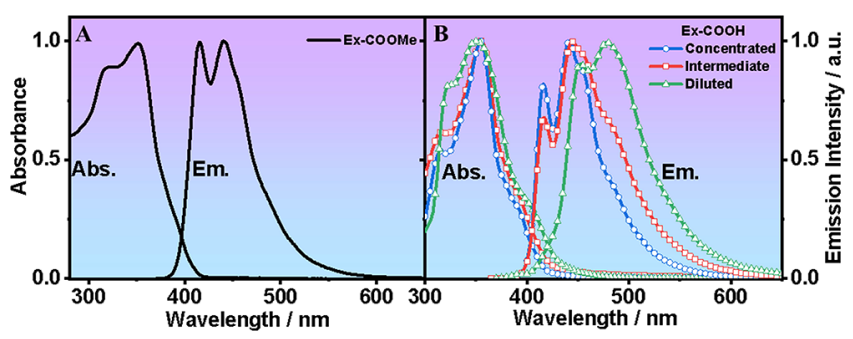

Figure 3. UV-visible absorption and emission spectra of (A) ExCOOMe and (B) Ex-COOH at different concentrations in DMF solutions. The excitation wavelength was $350 \mathrm{~nm}$. For diluted, intermediate, and concentrated samples of $\mathrm{Ex}-\mathrm{COOH}$, the ODs at $355 \mathrm{~nm}$ are $0.05,0.35$, and 1.78 , respectively.

390 The absorption spectra of both molecules are very similar in 391 shape, having an absorption band with two intensity maxima at 392320 and $350 \mathrm{~nm}$. We suggest that these bands reflect the $S_{0} \rightarrow$ $393 S_{1}(350 \mathrm{~nm})$ and $S_{0} \rightarrow S_{2}(320 \mathrm{~nm})$ transitions. Previously, 394 similar DBA derivatives, Nu-T12, T12-COOMe, and T12$395 \mathrm{COOH}$, have been investigated. ${ }^{56}$ The structural difference 396 between these molecules and those reported in this study is 397 shown in Scheme S2. The UV-visible absorption spectrum of $398 \mathrm{Nu}-\mathrm{T} 12$ in a DMF solution was characterized by an intensity 399 maximum at $291 \mathrm{~nm}$ assigned to the $S_{0} \rightarrow S_{4}$ transition, while 400 the $S_{0} \rightarrow S_{1,2,3}$ transitions are symmetry-forbidden. However, 401 the absorption spectra of T12-COOMe and $\mathrm{T} 12-\mathrm{COOH}$ in 402 DMF solutions displayed a maximum intensity at $325 \mathrm{~nm}$ 403 assigned to the $S_{0} \rightarrow S_{2}$ transition. ${ }^{56}$ Based on this, we suggest 404 that the ampliation of the core causes a loss of symmetry of the 405 molecule, thus allowing the $S_{0} \rightarrow S_{1}$ transition located at 350 $406 \mathrm{~nm}$ for both $\mathrm{Ex}-\mathrm{COOMe}$ and $\mathrm{Ex}-\mathrm{COOH}$, while the peak at $407320 \mathrm{~nm}$ reflects the $S_{0} \rightarrow S_{2}$ transition. As we have discussed in 408 the theoretical part, this molecule displays a high oscillator 409 strength of both transitions, suggesting that they are permitted 410 and visible in the absorption spectrum. It is worth mentioning 411 that, while the absorption spectrum of Ex-COOMe does not 412 depend on the used concentration, the one of Ex-COOH 413 presents some changes reflected in the intensity of the $320 \mathrm{~nm}$ 414 band. In particular, upon increasing the concentration, the 320 $415 \mathrm{~nm}$ band decreases in intensity, while the shoulder at $400 \mathrm{~nm}$ 416 slightly increases in intensity (Figure 3B). As we have 417 discussed in the theoretical part, the molecules of DMF 418 interact with the acid groups of $\mathrm{Ex}-\mathrm{COOH}$, generating new 419 complexed and stabilized structures, which can exist at the 420 ground state. Moreover, we have reported that similar 421 molecules with carboxylic acid groups in the extremes interact with DMF, generating $\mathrm{H}$-bonded complexes and anionic 422 species. ${ }^{47,56}$ Thus, we suggest that the absorption at $400 \mathrm{~nm}$ is 423 due to anionic structures as a result of dilution of Ex-COOH 424 weak acid.

425

The emission spectrum of Ex-COOMe in a DMF solution is 426 independent on the excitation wavelength and presents two 427 intensity maxima at 415 and $440 \mathrm{~nm}$. The full width at half 428 maximum (FWHM) of intensity is large $\left(3520 \mathrm{~cm}^{-1}\right), 429$ suggesting that the emission comes from more than one 430 species. The large Stokes shift is $\sim 5000 \mathrm{~cm}^{-1}$, suggesting the 431 occurrence of a reaction in the excited structures. Previously, 432 we assigned this reaction in T12-COOMe to an ICT event, 433 and we believe that it is also happening in excited Ex-COOMe. 434 Moreover, the theoretical calculations on $\mathrm{Ex}-\mathrm{COOH}$ suggest 435 the possibility of an ICT reaction in its excited state; hence, the 436 same reaction can occur in the methyl ester partner. The 437 emission spectra of Ex-COOH in DMF solutions exhibit a 438 dependence on its concentration (Figure 3B). For the 439 concentrated sample, the spectrum displays intensity maxima 440 at 415 and $440 \mathrm{~nm}$, like its ester methylated derivative, a 441 FWHM of $3300 \mathrm{~cm}^{-1}$, and a Stokes shift of $5500 \mathrm{~cm}^{-1}$. This 442 suggests that the electronic structures of the emitting species 443 are not very different, and the ICT reaction takes place at the 444 $\mathrm{S}_{1}$ states. However, when the concentration of $\mathrm{Ex}-\mathrm{COOH}$ is 445 very weak, the band around $415 \mathrm{~nm}$ vanishes, while a new 446 band at $480 \mathrm{~nm}$ appears. Based on the behavior of the 447 absorption spectrum of Ex-COOH upon dilution (formation of 448 anions), we suggest that this emission band at $480 \mathrm{~nm}$ is due to 449 the emission of anionic species. To support this assignment, we 450 added $100 \mu \mathrm{L}$ of $0.1 \mathrm{M} \mathrm{NaOH}$ (in DMF) to $3 \mathrm{~mL}$ of a 451 concentrated solution of Ex-COOH in DMF (Figure S2A). 452 The emission spectral shape of the concentrated sample turns 453 immediately to the one obtained for the diluted sample, 454 confirming that the anions are the emitting structures at low 455 concentrations. Moreover, taking into account the real signal of 456 the emission spectra, we can observe that the fluorescence of 457 the anionic species is less intense than the one of the ICT 458 species (Figure S2B).

To further characterize the spectroscopy of Ex-COOMe and 460 $\mathrm{Ex}-\mathrm{COOH}$ molecules, we have recorded their excitation 461 spectra in DMF solutions (Figure S3). The spectrum of Ex- 462 COOMe is quite similar in maxima and shape to the 463 absorption one, indicating a single ground state origin for 464 the excited species. However, the excitation spectrum of the 465 concentrated sample of Ex-COOH shows a red shift of the 466 intensity maximum compared to the absorption one when the 467 observation wavelength increases. This fact suggests that the 468 red-emitting species absorb in this region, and we assign them 469 to anions. The spectrum of $\mathrm{Ex}-\mathrm{COOH}$ at an intermediate 470 concentration is practically identical in shape to the absorption 471 one in the 420-600 $\mathrm{nm}$ observation range. However, 472 observing at $400 \mathrm{~nm}$ (the beginning of the emission spectrum), 473 the diluted sample displays a different spectrum whose 474 intensity maximum is blue-shifted with respect to the 475 absorption one. These species are the ones having established 476 H-bonds with DMF molecules. In brief, the steady-state 477 observations are in agreement with the previous theoretical 478 calculations: (i) the $S_{0} \rightarrow S_{1}$ transition is allowed due to the 479 asymmetry of the molecule. (ii) Different species co-exist at $S_{0} 480$ as a result of the interactions between the acid groups of Ex- 481 $\mathrm{COOH}$ and DMF molecules. (iii) An ICT reaction may occur 482 at $S_{1}$. 
484 3.3. Picosecond Time-Resolved Experiments in 485 Solution. To characterize the ps-ns dynamics and relaxation 486 of Ex-COOMe and Ex-COOH in DMF solutions, we carried 487 out ps experiments exciting at $371 \mathrm{~nm}$ and observing at 488 different emission wavelengths (Figure 4).
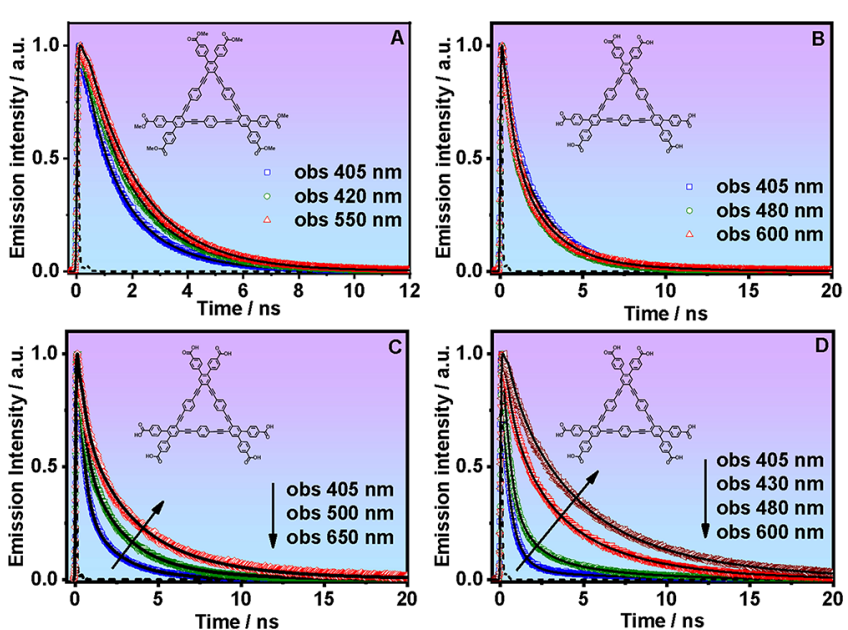

Figure 4. Magic-angle emission decays of (A) Ex-COOMe, (B) concentrated, (C) intermediate, and (D) diluted samples of Ex$\mathrm{COOH}$ in DMF solutions upon excitation at $371 \mathrm{~nm}$ and observation as indicated in the inset. The solid lines are from the best global fits using a multiexponential function. The dashed line is the IRF of the setup. For diluted, intermediate, and concentrated samples of Ex$\mathrm{COOH}$ in DMF solutions, the ODs at $355 \mathrm{~nm}$ were $0.05,0.35$, and 1.78 , respectively.

489 Figure 4A shows selected emission decays of Ex-COOMe in 490 DMF, which exhibit a biexponential behavior with time 491 constants of 940 ps and 2.02 ns (Table 1). Both components 492 are decaying at the observation range. While the contribution 493 of the shortest component decreases at longer wavelengths, the $494 \mathrm{~ns}$ one displays the opposite behavior. Based on the large 495 Stokes shift value $\left(5000 \mathrm{~cm}^{-1}\right)$, we suggested that an ICT 496 process is occurring at the $S_{1}$ state. Therefore, we assign the 497 shortest component to the emission of the initially excited 498 species, while the longest one is due to the formed ICT 499 species. Both theoretical calculations and previous studies of 500 comparable molecules support this assignment. ${ }^{33,56}$ The 501 difference between Ex-COOMe and the previously studied 502 one, T12-COOMe, is the ampliation of the main core in the 503 former with three benzenes rings and three triplet bonds (for 504 more details, see Scheme S2). ${ }^{56}$ This structural change 505 drastically modifies the photobehavior of the molecule because 506 the presence of benzene inhibits the emission from the triplet, 507 being therefore only detectable the fluorescence of the ICT 508 species.

509 Figure 4B-D displays selected emission decays of Ex$510 \mathrm{COOH}$ in DMF solutions at different concentrations, while 511 Table 1 shows the results of the multiexponential fits. The 512 decays of the concentrated sample practically do not change 513 with the observation wavelengths (Figure 4B). However, the 514 ones of the intermediate and diluted samples become longer 515 when observed at longer wavelengths. For all the studied 516 concentrations, we obtained a multiexponential behavior with 517 time constants of $\sim 400 \mathrm{ps,} \sim 2.2$, and $\sim 5.8 \mathrm{~ns}$. Nevertheless, 518 the contribution of each component to the global signal 519 depends on the sample concentration. For the most concentrated one, the contribution of the shortest component 520 ( $\sim 400$ ps) over the whole observation range has a value of 521 $\sim 40 \%$, while the one for the $2.2 \mathrm{~ns}$ component is $\sim 55 \%$. The 522 $5.8 \mathrm{~ns}$ component only appears at the reddest part of the 523 emission spectrum with a small contribution of $7 \%$. For the 524 intermediate and diluted samples, the contribution of the 525 shortest component decreases as we move to longer wave- 526 lengths, while the one of the $5.8 \mathrm{~ns}$ lifetime clearly increases. 527 The 2.2 ns component displays its maximum contribution at 528 $550 \mathrm{~nm}$. When the concentration increases, the contribution of 529 the intermediate becomes more noticeable, being this behavior 530 typical of aggregates. Moreover, being the $2.2 \mathrm{~ns}$ lifetime 531 similar to that obtained for Ex-COOMe (2.0 ns of ICT 532 species), we suggest that it is due to the emission of aggregates 533 with an ICT character. This fact is also supported by the large 534 Stokes shift observed from steady-state experiments, as Ex- 535 COOMe. The 400 ps component corresponds to Ex-COOH 536 structures H-bonded to DMF molecules, which can (i) relax to 537 $\mathrm{S}_{0}$ with a lifetime approximately of $400 \mathrm{ps}$, (2) undergo an ICT 538 reaction, or (3) suffer a proton transfer (PT) reaction to 539 generate the anionic species, which emits in the reddest region 540 with an emission lifetime of $5.8 \mathrm{~ns}$. Because the anions are also 541 present at $S_{0}$, their excitation generates a population of excited 542 anions within the laser pulse, and as a consequence, the rise 543 time, which should be observed as a result of PT events upon 544 excitation of $\mathrm{H}$-bonded Ex-COOH to DMF, cannot be 545 recorded. The fact that the contribution of the shortest 546 component does not decrease at longer observation wave- 547 lengths suggests the occurrence of an additional process, 548 generating the third, longest component $(5.82 \mathrm{~ns})$ due to the 549 anions and which are not formed in Ex-COOMe (Table 1). 550 The increase in the contribution of this component with 551 dilution (Ostwald's law) supports its assignment to anionic 552 species.

To further characterize the ps dynamics, we also carried out 554 ps time-resolved emission spectra (TRES) of all the samples 555 (Figure 5). The not normalized TRES are shown in Figure S5. 556 fs

The TRES of Ex-COOMe are similar to the steady-state 557 one, exhibiting two intensity maxima at 420 and $450 \mathrm{~nm}$. In 558 agreement with the above ps discussion, we observed a small 559 red shift of the peak at $450 \mathrm{~nm}$ when the gating time increases, 560 reflecting the ICT process occurrence at $S_{1}$. For Ex-COOH, 561 the TRES of the concentrated sample is the same during the 562 whole gating time window (from 0 to $15 \mathrm{~ns}$ ), which is in 563 concordance with the ps decays. For the intermediate and 564 diluted sample solutions, the peak at $415 \mathrm{~nm}$ at short gating 565 times $(<200 \mathrm{ps})$ is affected by the Raman signal from the 566 solvent. At the beginning, the emission comes fundamentally 567 from the complexes with DMF and aggregates species, while at 568 longer times, the emission around $500 \mathrm{~nm}$ increases due to the 569 anions. Scheme 1 illustrates the observed behavior for emission $570 \mathrm{~s} 1$ decays at the different generated species for $\mathrm{Ex}-\mathrm{COOH}$ and $\mathrm{Ex}-571$ COOMe.

3.4. Nanosecond (ns) Flash Photolysis Studies in 573 Solution. It is well known that DBA derivatives display a rich 574 slow photodynamic due to the triplet states. ${ }^{56,63,64}$ To explore 575 to the slow relaxation of the Ex-COOH molecule, we carried 576 out ns flash photolysis upon exciting at $355 \mathrm{~nm}$ (OD at 355577 $\mathrm{nm}$ of 1.78). The selection of the observation wavelengths 578 $(425,520$, and $660 \mathrm{~nm})$ were based on previous experiments 579 on a similar molecule, T12-COOH. ${ }^{56}$ Figure $6 \mathrm{~A}$ shows the $580 \mathrm{f} 6$ selected transient absorption decays of the excited sample 581 
Table 1. Values of Time Constants $\left(\tau_{i}\right)$, Normalized (to 100) Pre-exponential Factors $\left(a_{i}\right)$, and Contributions $\left(c_{i}=\tau_{i} \times a_{i}\right)$ in the Signal Obtained from a Global Multiexponential Fit of the Emission Decays of Ex-COOMe and Different Concentrations of Ex-COOH in DMF Solutions ${ }^{a}$

\begin{tabular}{|c|c|c|c|c|c|c|c|c|c|c|}
\hline sample & $\lambda_{\text {obs }}(\mathrm{nm})$ & $\tau_{1}(\mathrm{ps}) \pm 70 \mathrm{ps}$ & $a_{1}$ & $c_{1}$ & $\tau_{2}(\mathrm{~ns}) \pm 0.2 \mathrm{~ns}$ & $a_{2}$ & $c_{2}$ & $\tau_{3}(\mathrm{~ns}) \pm 0.2 \mathrm{~ns}$ & $a_{3}$ & $c_{3}$ \\
\hline \multirow{6}{*}{ Ex-COOMe } & 405 & 940 & 56 & 37 & 2.02 & 44 & 63 & & & \\
\hline & 420 & & 24 & 13 & & 76 & 87 & & & \\
\hline & 445 & & 14 & 7 & & 86 & 93 & & & \\
\hline & 460 & & 6 & 3 & & 94 & 97 & & & \\
\hline & 500 & & 4 & 2 & & 96 & 98 & & & \\
\hline & 550 & & 3 & 2 & & 97 & 98 & & & \\
\hline \multirow[t]{6}{*}{ Ex-COOH concentrated } & 405 & 410 & 39 & 10 & 2.21 & 61 & 90 & 5.82 & & \\
\hline & 430 & & 39 & 10 & & 61 & 90 & & & \\
\hline & 450 & & 40 & 11 & & 60 & 89 & & & \\
\hline & 480 & & 41 & 12 & & 59 & 88 & & & \\
\hline & 550 & & 41 & 10 & & 57 & 75 & & 2 & 9 \\
\hline & 600 & & 42 & 13 & & 51 & 66 & & 7 & 21 \\
\hline \multirow[t]{7}{*}{$\mathrm{Ex}-\mathrm{COOH}$ intermediate } & 405 & 390 & 76 & 32 & 2.23 & 24 & 68 & 5.75 & & \\
\hline & 420 & & 75 & 32 & & 25 & 68 & & & \\
\hline & 450 & & 70 & 26 & & 29 & 64 & & 1 & 10 \\
\hline & 460 & & 65 & 22 & & 32 & 64 & & 3 & 14 \\
\hline & 500 & & 51 & 12 & & 42 & 61 & & 7 & 27 \\
\hline & 550 & & 42 & 8 & & 45 & 53 & & 13 & 39 \\
\hline & 600 & & 36 & 6 & & 43 & 42 & & 21 & 52 \\
\hline \multirow[t]{6}{*}{ Ex-COOH diluted } & 405 & 400 & 92 & 57 & 2.25 & 6 & 18 & 5.95 & 2 & 24 \\
\hline & 430 & & 80 & 35 & & 16 & 41 & & 4 & 24 \\
\hline & 450 & & 51 & 12 & & 33 & 43 & & 16 & 45 \\
\hline & 480 & & 37 & 6 & & 42 & 44 & & 21 & 50 \\
\hline & 550 & & 22 & 3 & & 41 & 32 & & 37 & 65 \\
\hline & 600 & & 20 & 2 & & 30 & 21 & & 50 & 77 \\
\hline
\end{tabular}

${ }^{a_{T}}$ The excitation was at $371 \mathrm{~nm}$. For diluted, intermediate, and concentrated samples of Ex-COOH in DMF solutions, the ODs at $355 \mathrm{~nm}$ were $0.05,0.35$, and 1.78 , respectively.
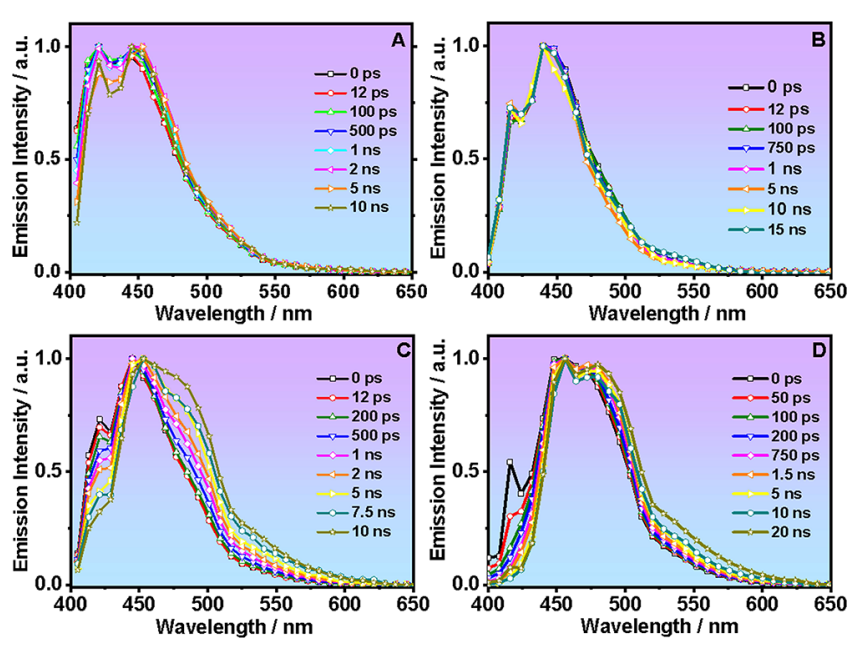

Figure 5. Normalized time-resolved emission spectra (TRES) of (A) Ex-COOMe, (B) concentrated, (C) intermediate, and (D) diluted samples of Ex-COOH in DMF solutions upon excitation at $371 \mathrm{~nm}$ and gating at the indicated delay times. For diluted, intermediate, and concentrated samples of Ex-COOH in DMF solutions, the ODs at $355 \mathrm{~nm}$ were $0.05,0.35$, and 1.78 , respectively. The emission at $\sim 420$ $\mathrm{nm}$ at the delay time $(<200 \mathrm{ps})$ is affected by the solvent Raman signal.

582 equilibrated in an air atmosphere. Table S6 shows the results 583 from the best fit of the experimental transient decays.

584 The fits of the decays at the three observation wavelengths $585(425,520$, and $660 \mathrm{~nm})$ give two time constants of $\sim 0.47$ and $586 \sim 2.0 \mu$ s (Table S6). The contribution of the longest
Scheme 1. Photophysical Diagram (Not in Scale) Showing the Electronic Transitions, Involved Events and Values of Their Time Constants of (A) Ex-COOMe and (B) Ex$\mathrm{COOH}$ in DMF Solutions
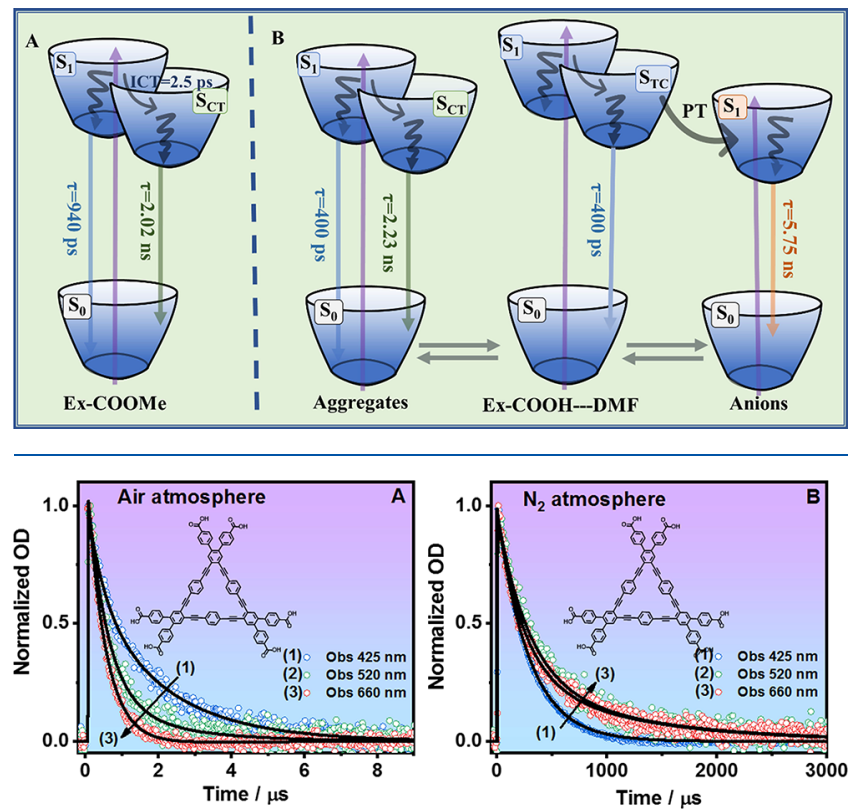

Figure 6. Selected transient absorption decays of Ex-COOH in DMF solution with an OD at 355 of 1.78 under (A) air and (B) nitrogen atmospheres and exciting at $355 \mathrm{~nm}$. 
587 component decreases at longer wavelengths, while the other 588 one displays the opposite behavior. We suggest that these two 589 states have the same nature, a triplet state. To corroborate this 590 assignment, we carried out experiments under nitrogen and 591 oxygen atmospheric conditions (Figure 6B and Figure S5, 592 respectively). As we can see, the decays become longer and 593 shorter under nitrogen and oxygen atmospheres, respectively, 594 than the ones under air conditions, confirming that the 595 involved states are $\mathrm{T}_{1}$. For the decays recorded under nitrogen, 596 we obtained time constants of 285 and $1140 \mu$ s. Based on the 597 contributions of both time constants, we suggest that the $2 \mu \mathrm{s}$ 598 (obtained under air conditions, and whose contribution 599 decreases at longer wavelengths) and the $0.45 \mu \mathrm{s}$ (obtained 600 under air conditions and whose contribution increases at 601 longer wavelengths) components correspond to the 285 and $6021140 \mu$ s components, respectively. The large increase between 603 the component under air $(0.45 \mu \mathrm{s})$ and nitrogen $(1140 \mu \mathrm{s})$ 604 atmospheres can be ascribed to very efficient interactions 605 (collisions and energy transfer) between this triplet state and 606 the oxygen molecule in DMF solution. The fits of the decays 607 under an oxygen atmosphere give values of $\sim 90 \mathrm{~ns}$ and $2 \mu \mathrm{s}$. 608 While the shortest component appears in the three observation 609 wavelengths; the longest one only appears at $425 \mathrm{~nm}$. We 610 suggest that the species with a time of $2 \mu$ s (which contributes 611 mainly in this observation wavelength) interacts strongly with 612 the oxygen molecule under air conditions; therefore, when we 613 add more oxygen, this triplet state is not affected. However, 614 when its contribution to the decay is very small (at 520 and $615660 \mathrm{~nm}$ ), the $0.45 \mu$ s component is largely affected by the 616 presence of oxygen, reflected as a short decay. Probably, this 617 time is a mixture of both state lifetimes.

618 3.5. Femtosecond Time-Resolved Experiments in ${ }_{619}$ Solution. To characterize the ultrafast photodynamics of 620 both molecules in DMF solutions, we recorded the femto621 second (fs) emission decays of Ex-COOMe and of Ex-COOH 622 in DMF upon excitation at $370 \mathrm{~nm}$ (Figure 7 and Figure S5). 623 While Figure 7A,B shows the decays at short time window,
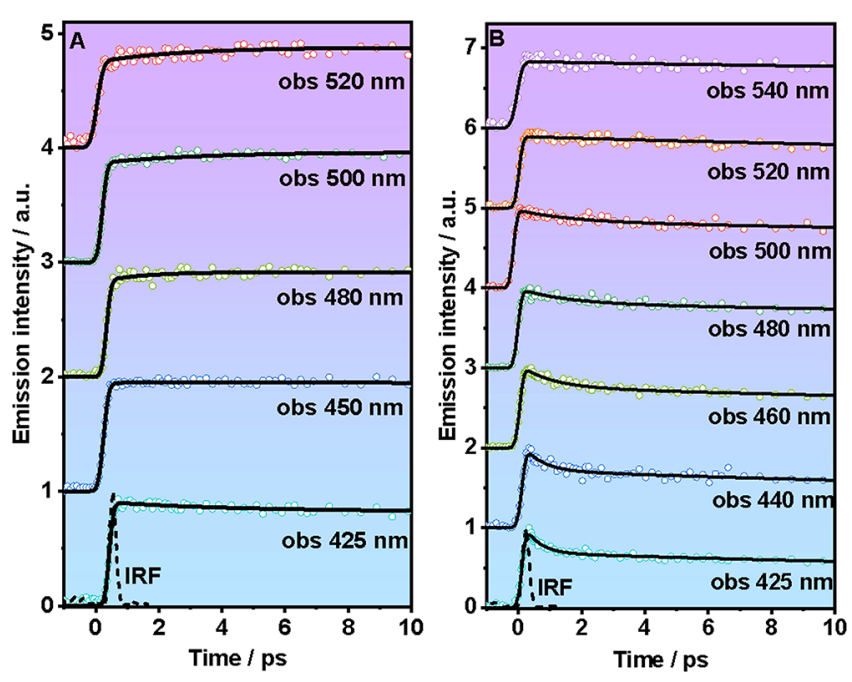

Figure 7. Representative fs emission transients in a short time window of (A) Ex-COOMe and (B) concentrated sample (OD $(355 \mathrm{~nm})=$ 1.78) of Ex-COOH in DMF solutions upon excitation at $370 \mathrm{~nm}$. The observation wavelengths are indicated in the inset. The solid lines are from the best fits using a multiexponential function, and IRF is the instrumental response function.
Figure S6 displays the ones at a longer one. Table 2 exhibits 624 t 2 the results from the best fits of the experimental decays.

Table 2. Values of Time Constants $\left(\tau_{i}\right)$ and Normalized (to 100) Pre-exponential Factors $\left(a_{i}\right)$ of the Function Used in Fitting the fs Emission Transients of Ex-COOMe and a Concentrated Sample (OD $(355 \mathrm{~nm})=1.78)$ of Ex-COOH in DMF Solutions upon Excitation at $370 \mathrm{~nm}^{a}$

\begin{tabular}{|cccccc} 
sample & $\begin{array}{c}\lambda_{\text {obs }} \\
(\mathrm{nm})\end{array}$ & $\begin{array}{c}\tau_{1}(\mathrm{ps}) \pm 0.5 \\
\mathrm{ps}\end{array}$ & \multicolumn{1}{c}{$a_{1}$} & $\tau_{2}(\mathrm{ps})$ & $a_{2}$ \\
\hline Ex-COOMe & 425 & 2.02 & 7 & $950^{*}$ & 93 \\
& 450 & & & & 100 \\
& 480 & 2.18 & -100 & & 100 \\
& 500 & 2.49 & -100 & & 100 \\
& 520 & 2.70 & -100 & & 100 \\
Ex-COOH & 425 & 3.15 & 17 & $400^{*}$ & 83 \\
concentrated & 440 & 3.10 & 14 & & 86 \\
& 460 & 3.20 & 14 & & 86 \\
& 480 & 3.31 & 13 & & 87 \\
& 500 & 3.42 & 8 & & 92 \\
& 520 & & & & 94 \\
& 540 & & & & 90
\end{tabular}

${ }^{a}$ The negative sign for $a_{i}$ indicates a rising component in the emission signal. The asterisk indicates a fixed value in the fit.

The fs emission decays of Ex-COOMe exhibit a 626 biexponential behavior with time constants of $2-2.7$ ps and 627 950 ps. The shortest component is decaying at the bluest part 628 of the emission spectrum and rising from $480 \mathrm{~nm}$. The longest 629 component is fixed in the fit using the value obtained in the ps 630 experiments. It is well known that vibrational cooling (VC) 631 and solvation of similar molecules in solution occur in the fs-ps 632 regime. $^{65-67}$ In addition to that, we have suggested in the 633 previous parts that Ex-COOMe undergoes an ICT reaction. 634 The rising component of 2.5 ps can also reflect this event. 635 Hence, the 2.5 ps time should be a combination of VC, 636 solvation, and ICT reactions.

For $\mathrm{Ex}-\mathrm{COOH}$ in DMF, we were able to carry out fs 638 experiments only using the concentrated sample due to the 639 impossibility to obtain clear decays from diluted samples. The 640 best fits of the experimental data in the 425-500 nm 641 observation range reveal a short decay time of 3.1-3.4 ps 642 and a longer one of $400 \mathrm{ps}$ fixed in the fit using the ps data 643 (Figure 7B and Table 2). The short component has a larger 644 contribution than that found for the transients of Ex-COOMe, 645 suggesting the occurrence of a short additional event in the 646 former. As we discussed above, Ex-COOH in DMF can 647 undergo a PT reaction producing anions. Thus, the short 648 component may comprise VC, solvation, ICT, and PT 649 reactions. Unfortunately, for wavelengths longer than 520650 $\mathrm{nm}$, the decay did not show any rise, which could be directly 651 assigned to the presence of ICT and PT reactions. One reason 652 of this lack in the recorded signals could be the presence of fast 653 decays (different that VC and ICT) of the anions at longer 654 wavelengths cancelling the expected rise in this region. 655

Scheme 1 summarizes the unraveled photodynamics of both 656 molecules in DMF solutions. Upon excitation at $370 \mathrm{~nm}, \mathrm{Ex}-657$ COOMe undergoes an ICT reaction in $2.5 \mathrm{ps}$, which produces 658 ICT species with a fluorescence lifetime of 2.02 ns. The 659 fluorescence lifetime of the initially excited species is 940 ps. 660 On the other hand, a diluted sample of the formed H-bonded 661 complexes undergoes ICT and PT reactions ( $\sim 3$ ps $), 662$ 
663 generating ICT species and anions, which display fluorescence 664 lifetimes of 400 ps and $5.8 \mathrm{~ns}$, respectively. In addition to this, 665 we also suggest the existence of aggregates, which undergo the 666 ICT process $(\sim 3 \mathrm{ps})$, generating ICT species with a lifetime of 6672 ns.

668 Comparing the behaviors of Ex-COOMe and $\mathrm{Ex}-\mathrm{COOH}$ 669 with those of previously studied analogous molecules (T12$670 \mathrm{COOH}$ and $\mathrm{T} 12-\mathrm{COOMe}$ ) in $\mathrm{DMF}$, we observe that 671 expanding the core of the molecular units largely changes 672 the photobehavior of the spectrum. T12-COOMe shows 673 emission from the triplet state $(24.5 \mathrm{~ns})$, while Ex-COOMe 674 does not, however, emitting from an ICT state. The emission 675 of T12-COOH has its maximum intensity at $607 \mathrm{~nm}$, while Ex$676 \mathrm{COOH}$ displays it at $480 \mathrm{~nm}$. Both $\mathrm{T} 12-\mathrm{COOH}$ and $\mathrm{Ex}-$ $677 \mathrm{COOH}$ form $\mathrm{H}$-bonds with DMF molecules, leading to the 678 formation of the anions in $\mathrm{Ex}-\mathrm{COOH}$, but not observed in $679 \mathrm{~T} 12-\mathrm{COOH}$. This reflects a different acidity of the $\mathrm{COOH}$ 680 groups in both molecules.

681 3.6. Steady-State and Picosecond Time-Resolved 682 Emission in the Solid State. After exploring the photo683 behavior of the fundamental molecular units of Ex-COOMe 684 and $\mathrm{Ex}-\mathrm{COOH}$, we characterized their spectroscopy and 685 dynamics in the solid state, namely, Ex-ester and Ex-apo 686 (Figure 8).

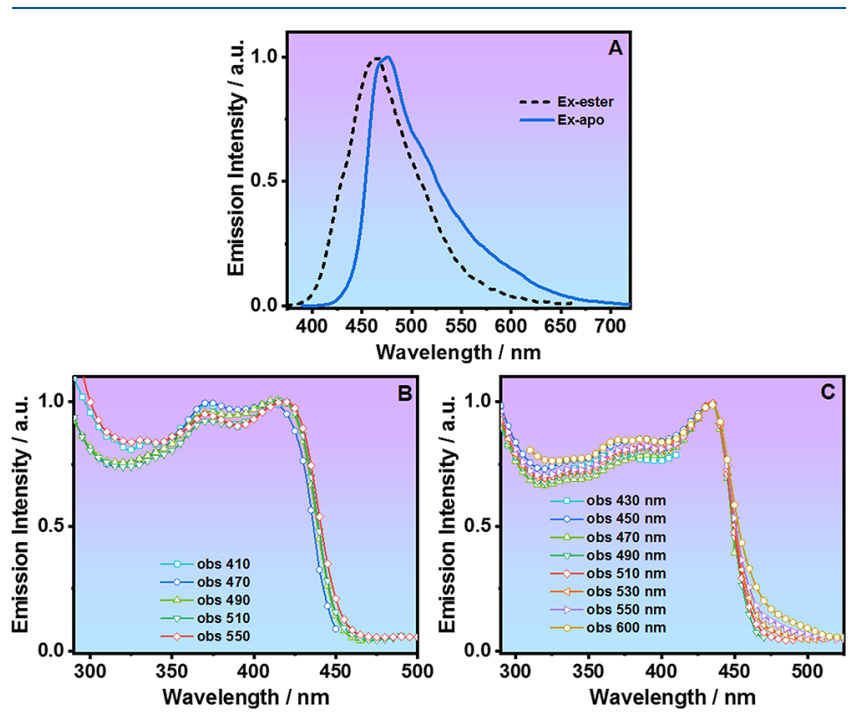

Figure 8. (A) Emission spectra of Ex-ester and Ex-apo in the solid state. The excitation wavelength was $380 \mathrm{~nm}$. (B, C) Excitation spectra of (B) Ex-ester and (C) Ex-apo in the solid state at the indicated observation wavelengths.

687 Figure 8A shows the recorded emission spectra of Ex-ester 688 and Ex-apo. While the former is constructed by the Ex689 COOMe molecules, Ex-apo, a HOF, is made by $\mathrm{H}$-bond 690 interactions between the Ex-COOH molecular units. Figure 1B 691 shows the network of the formed HOF and the interaction 692 between the acid groups of the Ex-COOH molecules. The 693 emission spectra of both Ex-ester and Ex-apo display intensity 694 maxima at 465 and $475 \mathrm{~nm}$, respectively. The excitation 695 spectra collected at different observation wavelengths are 696 similar, indicating a common ground-state origin of the 697 emitters (Figure 8B,C). The intensity maximum of the 698 excitation spectrum of Ex-ester is at $418 \mathrm{~nm}$, while that of 699 Ex-apo is at $435 \mathrm{~nm}$. This spectral shift of $930 \mathrm{~cm}^{-1}$ reflects 700 the effect of the H-bonds in the HOF (Figure 1). The smaller shift in emission at $450 \mathrm{~cm}^{-1}$ suggests the occurrence of a large 701 electronic change in Ex-ester.

To explore the photodynamics of the excited species in the 703 solid state, we carried out ps emission experiments on both 704 materials (Figure 9).
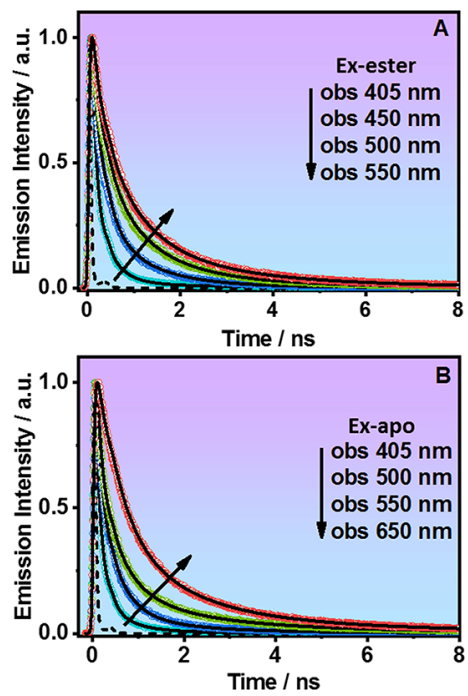

Figure 9. Magic-angle emission decays of (A) Ex-ester and (B) Exapo in the solid state upon excitation at $371 \mathrm{~nm}$ and recording at the indicated wavelengths. The solid lines are from the best global fits using a multiexponential function, and the dashed line is the IRF of the setup.

To begin with the Ex-ester sample, Figure 9A shows the 706 emission decays, and Table 3 gives the obtained results from 707 t3 the best global fits. We got emission lifetimes of 200 ps, 870 ps, 708 and $3.00 \mathrm{~ns}$. All the components are decaying over the 709 explored spectral range $(405-550 \mathrm{~nm})$. Clearly, the decays are 710 faster at shorter wavelengths and vanish at $\sim 2 \mathrm{~ns}$ after 711 excitation (Figure 8A). The shortest component presents its 712 maximum contribution (51\%) at the bluest part of the 713 spectrum, while the other two components increase their 714 contributions at the reddest one. Based on the results and 715 discussion of the molecular units in solution, we suggest that 716 the 200 ps component is due to the emission of the initially 717 excited species, while the other components are assigned to 718 different ICT structures generated after an ICT reaction. We 719 observed different lifetimes due to the heterogeneity of the 720 material and the possibility of having different conformers of 721 the methyl ester groups, leading to different characters of ICT 722 within the emitters. Note that the formed Ex-ester solid is 723 amorphous. Previously, we have reported on a comparable 724 material (T12-ester) in the solid state, observing two emission 725 lifetimes due to the formation of different CT species (5 and 726 $20 \mathrm{~ns})^{32,56}$

Figure 9B shows the emission decays of HOF Ex-apo in the 728 solid state upon excitation at $371 \mathrm{~nm}$, while Table 3 gives the 729 results from the best global fits. We obtained lifetimes of 100730 and 420 ps and 1.43 and 4.80 ns. We could not get and an 731 accurate fit using three components, as it was the case for the 732 Ex-ester sample. The decays are shorter at the blue side of the 733 emission spectrum, and no ps rising component was recorded. 734 While the shortest lifetime decreases its contribution at the 735 reddest part of the spectrum, the longer ones exhibit the 736 opposite behavior. The emission decays of both materials 737 
Table 3. Values of Time Constants $\left(\tau_{i}\right)$, Normalized (to 100) Pre-exponential Factors $\left(a_{i}\right)$, and Contributions $\left(c_{i}=\tau_{i} \times a_{i}\right)$ in the Signal Obtained from a Global Multiexponential Fit of the Emission Decays of Ex-ester and Ex-apo in the Solid State ${ }^{a}$

\begin{tabular}{|c|c|c|c|c|c|c|c|c|c|c|c|c|c|}
\hline sample & $\lambda_{\mathrm{obs}}(\mathrm{nm})$ & $\tau_{1}(\mathrm{ps}) \pm 30 \mathrm{ps}$ & $a_{1}$ & $c_{1}$ & $\tau_{2}(\mathrm{ps}) \pm 70 \mathrm{ps}$ & $a_{2}$ & $c_{2}$ & $\tau_{3}(\mathrm{~ns}) \pm 0.2 \mathrm{~ns}$ & $a_{3}$ & $c_{3}$ & $\tau_{4}(\mathrm{~ns}) \pm 0.3 \mathrm{~ns}$ & $a_{4}$ & $c_{4}$ \\
\hline \multirow[t]{7}{*}{ Ex-ester } & 405 & 200 & 80 & 51 & 870 & 19 & 41 & 3.00 & 1 & 8 & & & \\
\hline & 425 & & 78 & 48 & & 21 & 43 & & 1 & 9 & & & \\
\hline & 450 & & 73 & 35 & & 25 & 51 & & 2 & 14 & & & \\
\hline & 475 & & 60 & 21 & & 35 & 56 & & 4 & 23 & & & \\
\hline & 500 & & 55 & 17 & & 38 & 52 & & 7 & 31 & & & \\
\hline & 525 & & 50 & 14 & & 40 & 50 & & 10 & 36 & & & \\
\hline & 550 & & 44 & 11 & & 43 & 46 & & 13 & 43 & & & \\
\hline \multirow[t]{7}{*}{ Ex-apo } & 430 & 100 & 84 & 50 & 420 & 14 & 34 & 1.43 & 2 & 16 & 4.80 & & \\
\hline & 450 & & 74 & 36 & & 27 & 52 & & 2 & 12 & & & \\
\hline & 475 & & 66 & 28 & & 30 & 53 & & 4 & 19 & & & \\
\hline & 500 & & 60 & 20 & & 33 & 44 & & 5 & 24 & & 1 & 12 \\
\hline & 550 & & 52 & 11 & & 33 & 27 & & 12 & 34 & & 3 & 28 \\
\hline & 600 & & 25 & 3 & & 42 & 19 & & 27 & 44 & & 6 & 34 \\
\hline & 650 & & & & & 58 & 23 & & 35 & 46 & & 7 & 31 \\
\hline
\end{tabular}

${ }^{a_{T}}$ The excitation was at $371 \mathrm{~nm}$.

738 exhibit the same trend, and the lifetimes of the three short 739 components of the HOF have half values of those of the Ex740 ester, suggesting that the radiative rate constant in the former 741 are twice higher than in the latter (supposing that the radiative 742 lifetimes are the same for both materials). In addition to this 743 general trend, the decays of the former show a $4.80 \mathrm{~ns}$ 744 component at the green-red spectral region. Based on the 745 molecular structure of the HOF and on the discussion of the 746 involved $\mathrm{H}$-bonds units in the photodynamics of the molecular 747 units of the HOF, we assign this lifetime to the anions emitters. 748 Following the same scenario for Ex-ester, we assign the 400 ps 749 component to the emission of the initially excited molecular 750 units, while the intermediate ones ( 420 ps and $1.43 \mathrm{~ns}$ ) could 751 be due to the fluorescence of different ICT species. Previous 752 reports on the photobehavior of HOFs compared to those of 753 their amorphous partners support our assignment. ${ }^{32,34,48}$ 754 Scheme 2 illustrates the observed results and related 755 assignments for both Ex-ester and Ex-apo photobehaviours 756 in the solid state. Because the time resolution of the ps setup is 757 about 15 ps, we believe that the ICT events in both materials 758 are shorter than this time.

Scheme 2. Photophysical Diagram (Not in Scale) Showing the Electronic Transitions, Involved Events and Values of Their Time Constants of (A) Ex-ester and (B) Ex-apo in the Solid State ${ }^{a}$

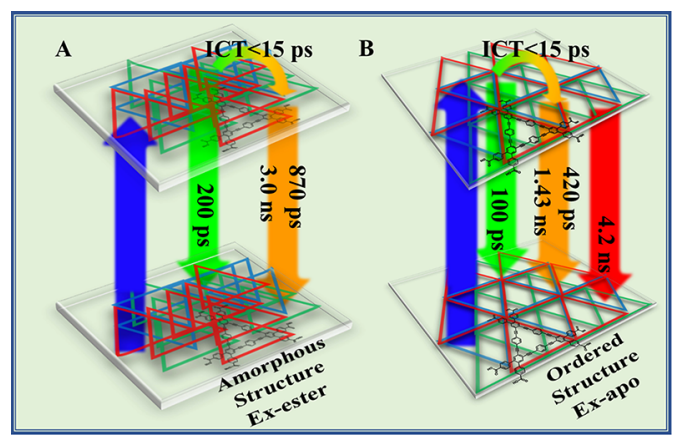

${ }^{a}$ The green arrows are from fluorescence of the initially excited species, the orange ones from the ICT species, while the red one is from the anionic species of HOF Ex-apo.
3.7. Confocal Microscopy Fluorescence and Time- 759 Resolved Studies. To explore the effect of possible defects 760 and orientation of the molecular units on the photodynamics 761 of this HOF, we performed ps confocal fluorescence 762 microscopy studies of single crystalline particles of Ex-apo 763 (Figure 10). $764 \mathrm{f} 10$
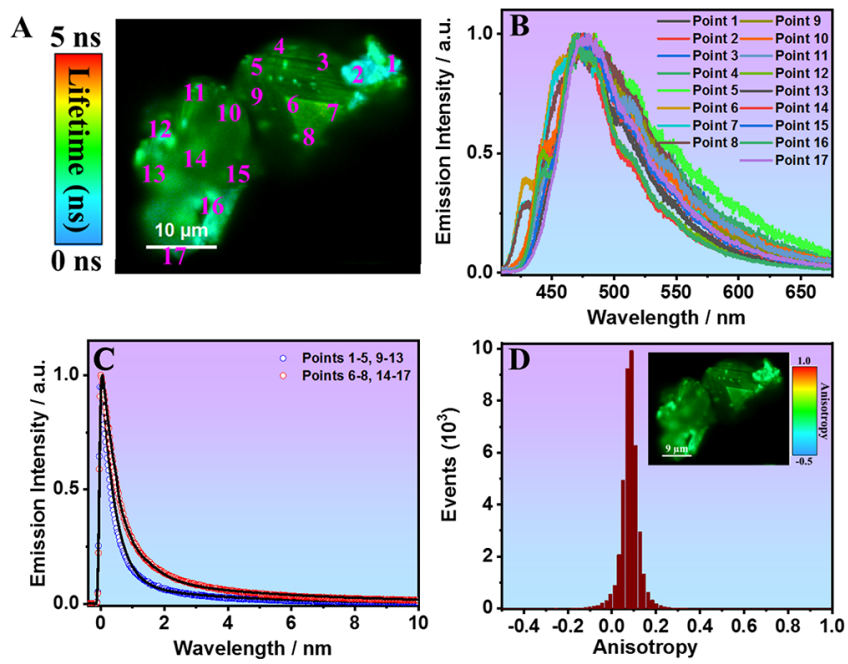

Figure 10. (A) FLIM, (B) emission spectra at different points, and (C) emission decays at the selected spectral range using a $460 \mathrm{~nm}$ long-pass filter of unwashed Ex-apo crystals. The solid lines are from the best fit using a multiexponential function. (D) Histogram of the emission anisotropy. The excitation wavelength was $370 \mathrm{~nm}$.

Figure 10A shows the fluorescence image (FLIM) of crystals 765 of around $15 \mu \mathrm{m}$ in length. From the color distribution, a large 766 green with a very small contribution of blue, we suggest that 767 the fluorescence lifetimes are the same over the crystals. In the 768 blue region, the contribution of the shortest fluorescence 769 lifetime is larger than in the green one. Figure 10B displays the 770 emission spectra at different selected points of the same 771 crystals. We observe that the intensity of the peak at $\sim 425 \mathrm{~nm} 772$ and the FWHM of the emission band change with the 773 recording position, possibility due to chemical or physical 774 defects on the crystals. Other crystals have been interrogated 775 
776 (Figures S7 and S8) and show a similar behavior, in agreement 777 with to other HOF spectroscopic studies. ${ }^{34,48}$

778 To shed more light on this behavior, ps time-resolved 779 emission experiments on the same points of the crystals were 780 carried out (Figure 10C). The results show a small difference 781 between the explored points of the crystal. Table S6A displays 782 the data from the best fits, while Table S7A shows a summary 783 of the obtained time constants and pre-exponential factor. 784 Practically, all the points exhibit a biexponential behavior with 785 time constants of 400 ps $(90 \%)$ and 3 ns (10\%), although 786 there are few zones where these time values increase to 600 ps 787 (90\%) and $4.5 \mathrm{~ns}(10 \%)$. Based on the ps experiments on bulk 788 and ensemble of crystals, we suggest that the longest 789 fluorescence lifetime is attributed to species having H-bond 790 interactions, while the shortest one could be affected by 791 (physical or chemical) defects or CT species. Physical defects 792 can be observed in the SEM image as cracking in the crystals. 793 In Figure 1C, we can see crystals with different shapes and 794 lengths. It is worth mentioning that large crystals also display 795 attached or adsorbed to their surface small ones, making the 796 heterogeneity of the system complex.

797 To eliminate these small and adsorbed crystals on the large 798 ones, we washed the sample with diethyl ether (Figure 11) and 799 acetone (Figure S9).
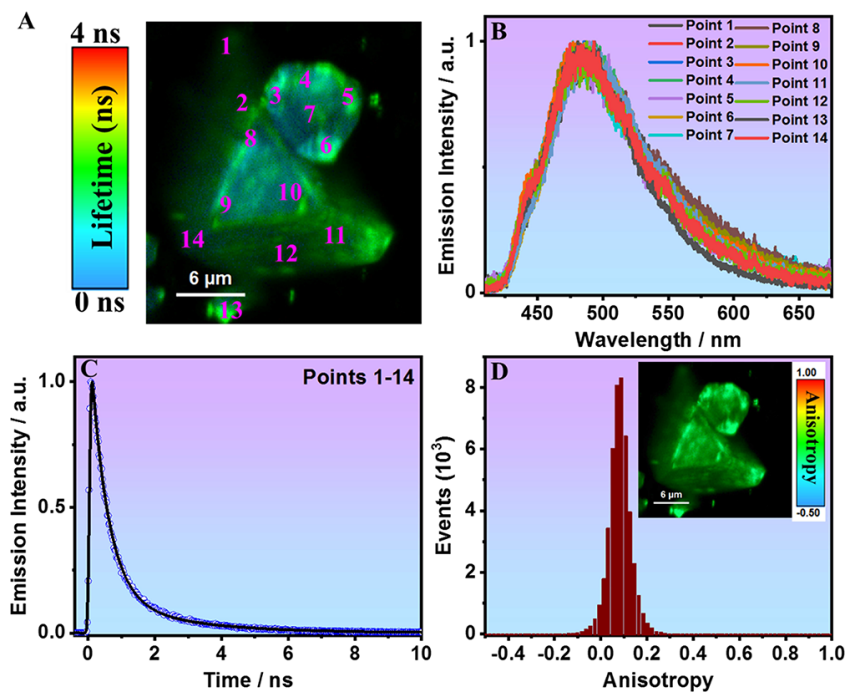

Figure 11. (A) FLIM, (B) emission spectra at different points, and (C) emission decays at the selected spectral range using a $460 \mathrm{~nm}$ long-pass filter of Ex-apo crystals washed with diethyl ether. The solid lines are from the best fit using a multiexponential function. (D) Histogram of the emission anisotropy. The excitation wavelength was $370 \mathrm{~nm}$.

800 Clearly, the emission spectra do not depend on the 801 interrogated site on the crystal, contrary to the behavior on 802 the unwashed ones (Figure 10B). Thus, upon washing, we 803 could eliminate small crystals and possibly molecules adsorbed 804 or adhered on the surface of the large crystals. The emission 805 decays of the washed crystals do not depend on the 806 interrogated point (Figure 11C and Figure S9C). Therefore, 807 the observed behavior in the unwashed crystals (peak at 425 $808 \mathrm{~nm}, \mathrm{FWHM}$, and different lifetimes) are not due to intrinsic 809 defects on the crystals. The fit of the decays shows a 810 biexponential behavior with time constants of 500-580 ps 811 (85-94\%) and 2.4-3.0 ns (15-6\%) (Table S7). To make an easy comparison between the results of the unwashed and 812 washed crystals, Table S8B shows a summary of the time 813 constants and their contributions. The absence of the $4.5 \mathrm{~ns} 814$ component, which appeared in the unwashed crystals, leads us 815 to suggest that the solvent has interpenetrated in the HOF 816 network (probably in the H-bonds) by frustrating these 817 connections, causing the lack of species having a H-bond 818 character. This fact is also supported by numerous articles 819 explaining that, during the crystallization of the HOFs, the 820 solvent interpenetrates in the H-bonds, thus frustrating the 821 right position of the molecular units to make the net- 822 work. $9,49,68-70$

823

To be able to study the anisotropy of the material, we 824 carried out fluorescence confocal microcopy measurements on 825 Ex-apo crystals, which show a high anisotropic emission 826 behavior (Figures 10D and 11D). The emission anisotropy 827 distribution displays one narrow band with the intensity 828 maximum around 0.1 . The extremely narrowed distribution 829 anisotropy suggests a large orientation of the emitters inside 830 the crystals. Previously, we have reported on other HOFs with 831 different fundamental units, displaying a large anisotropic 832 behavior. $^{32,33,48}$

Finally, comparing our previous report on HOF T12-apo ${ }_{834}^{32}$ with the present one (HOF Ex-apo), we observed that an 835 expansion of the core of the fundamental units, which leads to 836 an increase in the pore size of the HOF, modifies the 837 photobehavior. For T12-apo, we have observed that the 838 emission spectra of large crystals display a vibrational structure 839 due to the rigidity of the network and a long fluorescence 840 lifetime (22 ns). However, the emission spectrum of Ex-apo 841 does not exhibit such a vibrational structure, and its longest 842 fluorescence lifetime is 4.2 ns. These results can be explained 843 by the higher network flexibility of Ex-apo compared to that of 844 T12-apo, as a result of the expansion in the fundamental units 845 of the former one.

846

\section{CONCLUSIONS}

In this work, we have reported on studies of two phenylene- 847 ethynylene macrocycles: Ex-COOH and Ex-COOMe. The- 848 oretical calculations show that the Ex-COOH molecule could 849 exhibit an ICT at $S_{1}$ and that different complexes can co-exist 850 at the $S_{0}$ due to strong interactions between the acid groups 851 and the DMF molecules. Experimentally, Ex-COOMe in DMF 852 exists in one conformation, and upon excitation, we have 853 observed emissions from the initially excited state (940 ps) and 854 ICT species $(2.02 \mathrm{~ns})$ produced by an ICT reaction in $2.5 \mathrm{ps} .855$ For $\mathrm{Ex}-\mathrm{COOH}$ in DMF, we observed emissions of different 856 species, whose fluorescence lifetimes go from 400 ps to 5.95 ns. 857 Moreover, the ICT reaction is faster in $\mathrm{Ex}-\mathrm{COOH}(<200 \mathrm{fs}) 858$ than in Ex-COOMe. The ns flash photolysis experiments on 859 Ex-COOH indicate two different triplet states having lifetimes 860 of 0.45 and $2 \mu \mathrm{s}$. From the solid-state experiments, we 861 observed that both excited materials show fluorescence 862 lifetimes from 100 ps to 4.8 ns. Fluorescence microscopy 863 experiments on HOF Ex-apo single crystals indicate that they 864 do not exhibit a photobehavior dependent on the interrogated 865 site, while their emission anisotropy shows that the emitters are 866 well oriented in the crystals. We believe that the present 867 findings will trigger more research in the family of shape- 868 persistent phenylene-ethynylene macrocycles and related 869 HOFs for potential applications in photonics. 


\section{S1 Supporting Information}

873 The Supporting Information is available free of charge at 874 https://pubs.acs.org/doi/10.1021/acs.jpcc.0c01369.

875 Optimized molecular structure of $\mathrm{Ex}-\mathrm{COOH}$ interacting 876 with six molecules of DMF (Scheme S1), the structures 877 of the studied molecules similar to the ones of this 878 report (Scheme S2), the diagram of the energy level of $879 \mathrm{Nu}-\mathrm{Ex}$ and $\mathrm{Ex}-\mathrm{COOH}$ in DMF (Figure S1), the 880 absorption and emission spectra of Ex-COOH in DMF 881 solutions with $0.1 \mathrm{M}$ of $\mathrm{NaOH}$ (Figure S2), the 882 excitation spectra of Ex-COOMe and $\mathrm{Ex}-\mathrm{COOH}$ at 883 different concentrations in DMF (Figure S3), the not 884 normalized TRES of Ex-COOMe and $\mathrm{Ex}-\mathrm{COOH}$ in 885 DMF solutions (Figure S4), the ns- $\mu$ s transient 886 absorption decays of Ex-COOH (Figure S5), the fs 887 emission transient at a longer time window of Ex$888 \mathrm{COOMe}$ and the concentrated sample of $\mathrm{Ex}-\mathrm{COOH}$ in 889 DMF solutions (Figure S6), FLIM, emission spectra, 890 decays, and anisotropy of different crystals of Ex-apo 891 unwashed (Figures S7 and S8), a fluorescence 892 microscopy study of Ex-apo crystals washed with 893 acetone (Figure S9), the data of the internuclear 894 distance and the Mulliken charges of different electronic 895 states of $\mathrm{Nu}-\mathrm{Ex}$ and $\mathrm{Ex}-\mathrm{COOH}$ (Tables S1-S4), the 896 value of the relative energy stabilization of $\mathrm{Ex}-\mathrm{COOH}$ 897 interacting with DMF molecules (Table S5), the 898 obtained data from the fits of the transient absorption 899 decays of $\mathrm{Ex}-\mathrm{COOH}$ in DMF solutions under air, 900 oxygen, and nitrogen atmospheres (Table S6), and all 901 the obtained time constants and pre-exponential factors 902 used in the fit of the emission decays of Ex-apo single 903 crystals using a fluorescence microscope (Tables S7 and 904 S8) (PDF)

\section{AUTHOR INFORMATION}

\section{Corresponding Authors}

907 Ichiro Hisaki - Research Institute for Electronic Science,

908 Hokkaido University, Sapporo, Hokkaido 001-0020, Japan;

909 ๑ orcid.org/0000-0002-8170-5605; Email: hisaki@

$910 \quad$ es.hokudai.ac.jp

911 Abderrazzak Douhal - Departamento de Química Física,

912 Facultad de Ciencias Ambientales y Bioquimica, and INAMOL, 913 Universidad de Castilla-La Mancha, 45071 Toledo, Spain;

914 Email: abderrazzak.douhal@uclm.es

\section{Authors}

916 Eduardo Gomez - Departamento de Química Física, Facultad

917 de Ciencias Ambientales y Bioquímica, and INAMOL,

918 Universidad de Castilla-La Mancha, 45071 Toledo, Spain

919 Maria Rosaria di Nunzio - Departamento de Química Física,

920 Facultad de Ciencias Ambientales y Bioquimica, and INAMOL,

921 Universidad de Castilla-La Mancha, 45071 Toledo, Spain

922 Miquel Moreno - Universitat Autónoma de Barcelona,

923 Bellaterra 08193, Barcelona, Spain

924 Complete contact information is available at:

925 https://pubs.acs.org/10.1021/acs.jpcc.0c01369

\section{Author Contributions}

927 I.H. designed and made the materials. M.M. performed the 928 theoretical calculations and wrote the theoretical results. E.G. 929 and A.D. designed and carried out the experiments, analyzed, and discussed the theoretical and experimental results. E.G., 930 M.R.d.N., and A.D. wrote the manuscript. All the authors have 931 read the manuscript.

Notes

The authors declare no competing financial interest.

\section{ACKNOWLEDGMENTS}

This work was supported by the MINECO through project 936 MAT2017-86532-R and CTQ2017-83745-P and by KAKEN- 937 HI grant numbers JP18H01966 and JP19H04557 from MEXT, 938 Japan. E.G. thanks the MINECO for the Ph.D. fellowship. The 939 use of computational facilities at the "Consorci de Serveis 940 Universitaris de Catalunya (CSUC)" is gratefully acknowl- 941 edged.

\section{REFERENCES}

943

(1) Barbour, L. J. Crystal porosity and the burden of proof. Chem. 944 Commun. 2006, 11, 1163-1168.

(2) McKeown, N. B. Nanoporous molecular crystals. J. Mater. Chem. 946 2010, 20, 10588-10597.

(3) Holst, J. R.; Trewin, A.; Cooper, A. I. Porous organic molecules. 948 Nat. Chem. 2010, 2, 915-920.

(4) Tian, J.; Thallapally, P. K.; McGrail, B. P. Porous organic 950 molecular materials. CrystEngComm 2012, 14, 1909-1919. 951

(5) Mastalerz, M. Permanent Porous Materials from Discrete 952 Organic Molecules-Towards Ultra-High Surface Areas. Chem. - 953 Eur. J. 2012, 18, 10082-10091.

(6) Cooper, A. I. Porous Molecular Solids and Liquids. ACS Cent. 955 Sci. 2017, 3, 544-553.

956

(7) Luo, J.; Wang, J.-W.; Zhang, J.-H.; Lai, S.; Zhong, D.-C. 957 Hydrogen-bonded organic frameworks: design, structures and 958 potential applications. CrystEngComm 2018, 20, 5884-5898. 959

(8) Lin, R.-B.; He, Y.; Li, P.; Wang, H.; Zhou, W.; Chen, B. 960 Multifunctional porous hydrogen-bonded organic framework materi- 961 als. Chem. Soc. Rev. 2019, 48, 1362-1389.

(9) Hisaki, I.; Xin, C.; Takahashi, K.; Nakamura, T. Designing 963 Hydrogen-Bonded Organic Frameworks (HOFs) with Permanent 964 Porosity. Angew. Chem. Int. Ed. 2019, 58, 11160-11170. 965

(10) Huang, Q.; Li, W.; Mao, Z.; Qu, L.; Li, Y.; Zhang, H.; Yu, T.; 966 Yang, Z.; Zhao, J.; Zhang, Y.; et al. An exceptionally flexible hydrogen- 967 bonded organic framework with large-scale void regulation and 968 adaptive guest accommodation abilities. Nat. Commun. 2019, 10, 969 3074.

970

(11) Cui, Y.; Yue, Y.; Qian, G.; Chen, B. Luminescent Functional 971 Metal-Organic Frameworks. Chem. Rev. 2012, 112, 1126-1162. 972

(12) Abánades Lázaro, I.; Forgan, R. S. Application of zirconium 973 MOFs in drug delivery and biomedicine. Coord. Chem. Rev. 2019, 974 $380,230-259$. 975

(13) Zhou, H.-C. J.; Kitagawa, S. Metal-Organic Frameworks 976 (MOFs). Chem. Soc. Rev. 2014, 43, 5415-5418.

(14) Ding, M.; Flaig, R. W.; Jiang, H.-L.; Yaghi, O. M. Carbon 978 capture and conversion using metal-organic frameworks and MOF- 979 based materials. Chem. Soc. Rev. 2019, 48, 2783-2828. 980

(15) Cui, Y.; Zhang, J.; He, H.; Qian, G. Photonic functional metal- 981 organic frameworks. Chem. Soc. Rev. 2018, 47, 5740-5785.

(16) Feng, X.; Ding, X.; Jiang, D. Covalent organic frameworks. 983 Chem. Soc. Rev. 2012, 41, 6010-6022.

(17) Côté, A. P.; Benin, A. I.; Ockwig, N. W.; O’Keeffe, M.; Matzger, 985 A. J.; Yaghi, O. M. Porous, Crystalline, Covalent Organic Frameworks. 986 Science 2005, 310, 1166.

987

(18) Liu, X.; Huang, D.; Lai, C.; Zeng, G.; Qin, L.; Wang, H.; Yi, H.; 988 Li, B.; Liu, S.; Zhang, M.; et al. Recent advances in covalent organic 989 frameworks (COFs) as a smart sensing material. Chem. Soc. Rev 2019, 990 48, 5266-5302.

(19) Wang, H.; Zeng, Z.; Xu, P.; Li, L.; Zeng, G.; Xiao, R.; Tang, Z.; 992 Huang, D.; Tang, L.; Lai, C.; et al. Recent progress in covalent organic 993 
994 framework thin films: fabrications, applications and perspectives. 995 Chem. Soc. Rev. 2019, 48, 488-516.

996 (20) Rodríguez-San-Miguel, D.; Zamora, F. Processing of covalent 997 organic frameworks: an ingredient for a material to succeed. Chem. 998 Soc. Rev. 2019, 48, 4375-4386.

999 (21) Kreno, L. E.; Leong, K.; Farha, O. K.; Allendorf, M.; Van 1000 Duyne, R. P.; Hupp, J. T. Metal-Organic Framework Materials as 1001 Chemical Sensors. Chem. Rev. 2011, 112, 1105-1125.

1002 (22) Crowe, J. W.; Baldwin, L. A.; McGrier, P. L. Luminescent 1003 Covalent Organic Frameworks Containing a Homogeneous and 1004 Heterogeneous Distribution of Dehydrobenzoannulene Vertex Units. 1005 J. Am. Chem. Soc. 2016, 138, 10120-10123.

1006 (23) Ding, S.-Y.; Gao, J.; Wang, Q.; Zhang, Y.; Song, W.-G.; Su, C.1007 Y.; Wang, W. Construction of Covalent Organic Framework for 1008 Catalysis: Pd/COF-LZU1 in Suzuki-Miyaura Coupling Reaction. J. 1009 Am. Chem. Soc. 2011, 133, 19816-19822.

1010 (24) Dogru, M.; Handloser, M.; Auras, F.; Kunz, T.; Medina, D.; 1011 Hartschuh, A.; Knochel, P.; Bein, T. A Photoconductive Thienothio1012 phene-Based Covalent Organic Framework Showing Charge Transfer 1013 Towards Included Fullerene. Angew. Chem. Int. Ed. 2013, 52, 292010142924.

1015 (25) Sun, Z.; Li, Y.; Chen, L.; Jing, X.; Xie, Z. Fluorescent 1016 Hydrogen-Bonded Organic Framework for Sensing of Aromatic 1017 Compounds. Cryst. Growth Des. 2015, 15, 542-545.

1018 (26) Yu, T.; Ou, D.; Yang, Z.; Huang, Q.; Mao, Z.; Chen, J.; Zhang, 1019 Y.; Liu, S.; Xu, J.; Bryce, M. R.; et al. The HOF structures of 1020 nitrotetraphenylethene derivatives provide new insights into the 1021 nature of AIE and a way to design mechanoluminescent materials. 1022 Chem. Sci. 2017, 8, 1163-1168.

1023 (27) Han, Y.-F.; Yuan, Y.-X.; Wang, H.-B. Porous Hydrogen-Bonded 1024 Organic Frameworks. Molecules 2017, 22, 266.

1025 (28) Adachi, T.; Ward, M. D. Versatile and Resilient Hydrogen1026 Bonded Host Frameworks. Acc. Chem. Res. 2016, 49, 2669-2679.

1027 (29) Yang, W.; Yang, F.; Hu, T.-L.; King, S. C.; Wang, H.; Wu, H.; 1028 Zhou, W.; Li, J.-R.; Arman, H. D.; Chen, B. Microporous 1029 Diaminotriazine-Decorated Porphyrin-Based Hydrogen-Bonded Or1030 ganic Framework: Permanent Porosity and Proton Conduction. Cryst. 1031 Growth Des. 2016, 16, 5831-5835.

1032 (30) Hu, F.; Liu, C.; Wu, M.; Pang, J.; Jiang, F.; Yuan, D.; Hong, M. 1033 An Ultrastable and Easily Regenerated Hydrogen-Bonded Organic 1034 Molecular Framework with Permanent Porosity. Angew. Chem. Int. Ed. 1035 2017, 56, 2101-2104.

1036 (31) Karmakar, A.; Illathvalappil, R.; Anothumakkool, B.; Sen, A.; 1037 Samanta, P.; Desai, A. V.; Kurungot, S.; Ghosh, S. K. Hydrogen1038 Bonded Organic Frameworks (HOFs): A New Class of Porous 1039 Crystalline Proton-Conducting Materials. Angew. Chem. Int. Ed. 2016, 1040 55, 10667-10671.

1041 (32) Gomez, E.; Gutiérrez, M.; Cohen, B.; Hisaki, I.; Douhal, A. 1042 Single crystal fluorescence behavior of a new Hof material: A potential 1043 candidate for a new LED. J. Mater. Chem. C 2018, 6, 6929-6939.

1044 (33) Hisaki, I.; Ikenaka, N.; Gomez, E.; Cohen, B.; Tohnai, N.; 1045 Douhal, A. Hexaazatriphenylene-Based Hydrogen-Bonded Organic 1046 Framework with Permanent Porosity and Single-Crystallinity. Chem. 1047 - Eur. J. 2017, 23, 11611-11619.

1048 (34) Hisaki, I.; Suzuki, Y.; Gomez, E.; Ji, Q.; Tohnai, N.; Nakamura, 1049 T.; Douhal, A. Acid Responsive Hydrogen-Bonded Organic Frame1050 works. J. Am. Chem. Soc. 2019, 141, 2111.

1051 (35) Wang, H.; Li, B.; Wu, H.; Hu, T.-L.; Yao, Z.; Zhou, W.; Xiang, 1052 S.; Chen, B. A Flexible Microporous Hydrogen-Bonded Organic 1053 Framework for Gas Sorption and Separation. J. Am. Chem. Soc. 2015, 1054 137, 9963-9970.

1055 (36) Yin, Q.; Zhao, P.; Sa, R.-J.; Chen, G.-C.; Lü, J.; Liu, T.-F.; Cao, 1056 R. An Ultra-Robust and Crystalline Redeemable Hydrogen-Bonded 1057 Organic Framework for Synergistic Chemo-Photodynamic Therapy. 1058 Angew. Chem., Int. Ed. 2018, 57, 7691-7696.

1059 (37) Li, Z.; Xu, F.; Liu, Z.; Qin, C.; Ren, H.; Li, Y. Facile synthesis of 1060 novel porous self-assembling hydrogen-bonding covalent organic 1061 polymers and their applications towards fluoroquinolone antibiotics 1062 adsorption. RSC Adv. 2018, 8, 33516-33522.
(38) Tahara, K.; Yamamoto, Y.; Gross, D. E.; Kozuma, H.; Arikuma, 1063 Y.; Ohta, K.; Koizumi, Y.; Gao, Y.; Shimizu, Y.; Seki, S.; et al. 1064 Syntheses and Properties of Graphyne Fragments: Trigonally 1065 Expanded Dehydrobenzo[12] annulenes. Chem. - Eur. J. 2013, 19, 1066 11251-11260.

(39) Yoshimura, T.; Inaba, A.; Sonoda, M.; Tahara, K.; Tobe, Y.; 1068 Williams, R. V. Synthesis and Properties of Trefoil-Shaped Tris- 1069 (hexadehydrotribenzo[ 12 annulene) and Tris- 1070 (tetradehydrotribenzo[12] annulene). Org. Lett. 2006, 8, 2933-2936. 1071 (40) Johnson, C. A.; Lu, Y.; Haley, M. M. Carbon Networks Based 1072 on Benzocyclynes. 6. Synthesis of Graphyne Substructures via 1073 Directed Alkyne Metathesis. Org. Lett. 2007, 9, 3725-3728. 1074

(41) Hisaki, I.; Sakamoto, Y.; Shigemitsu, H.; Tohnai, N.; Miyata, 1075 M.; Seki, S.; Saeki, A.; Tagawa, S. Superstructure-Dependent Optical 1076 and Electrical Properties of an Unusual Face-to-Face, $\pi$-Stacked, One- 1077 Dimensional Assembly of Dehydrobenzo[12] annulene in the 1078 Crystalline State. Chem. - Eur. J. 2008, 14, 4178-4187. 1079

(42) Zentner, C. A.; Lai, H. W. H.; Greenfield, J. T.; Wiscons, R. A.; 1080 Zeller, M.; Campana, C. F.; Talu, O.; FitzGerald, S. A.; Rowsell, J. L. 1081 C. High surface area and $\mathrm{Z}^{\prime}$ in a thermally stable 8 -fold polycatenated 1082 hydrogen-bonded framework. Chem. Commun. 2015, 51, 11642- 1083 11645 .

1084

(43) Nandi, S.; Chakraborty, D.; Vaidhyanathan, R. A permanently 1085 porous single molecule $\mathrm{H}$-bonded organic framework for selective 1086 CO2 capture. Chem. Commun. 2016, 52, 7249-7252.

1087

(44) Alarcos, N.; Cohen, B.; Ziółek, M.; Douhal, A. Photochemistry 1088 and Photophysics in Silica-Based Materials: Ultrafast and Single 1089 Molecule Spectroscopy Observation. Chem. Rev. 2017, 117, 13639- 1090 13720.

1091

(45) Bian, L.; Shi, H.; Wang, X.; Ling, K.; Ma, H.; Li, M.; Cheng, Z.; 1092 Ma, C.; Cai, S.; Wu, Q.; et al. Simultaneously Enhancing Efficiency 1093 and Lifetime of Ultralong Organic Phosphorescence Materials by 1094 Molecular Self-Assembly. J. Am. Chem. Soc. 2018, 140, 10734-10739. 1095

(46) Wang, H.; Bao, Z.; Wu, H.; Lin, R.-B.; Zhou, W.; Hu, T.-L.; Li, 1096 B.; Zhao, J. C.-G.; Chen, B. Two solvent-induced porous hydrogen- 1097 bonded organic frameworks: solvent effects on structures and 1098 functionalities. Chem. Commun. 2017, 53, 11150-11153. 1099

(47) Gomez, E.; Suzuki, Y.; Hisaki, I.; Moreno, M.; Douhal, A. 1100 Spectroscopy and dynamics of a HOF and its molecular units: 1101 remarkable vapor acid sensing. J. Mater. Chem. C 2019, 7, 10818- 1102 10832.

1103

(48) Hisaki, I.; Suzuki, Y.; Gomez, E.; Cohen, B.; Tohnai, N.; 1104 Douhal, A. Docking Strategy to Construct Thermostable, Single- 1105 Crystalline, Hydrogen-Bonded Organic Framework with High Surface 1106 Area. Angew. Chem., Int. Ed. 2018, 57, 12650-12655.

1107

(49) Hisaki, I.; Nakagawa, S.; Ikenaka, N.; Imamura, Y.; Katouda, 1108 M.; Tashiro, M.; Tsuchida, H.; Ogoshi, T.; Sato, H.; Tohnai, N.; et al. 1109 A Series of Layered Assemblies of Hydrogen-Bonded, Hexagonal 1110 Networks of C3-Symmetric $\pi$-Conjugated Molecules: A Potential 1111 Motif of Porous Organic Materials. J. Am. Chem. Soc. 2016, 138, 1112 6617-6628.

1113

(50) Handy, N. C. The molecular physics lecture 2004: (i) Density 1114 functional theory, (ii) Quantum Monte Carlo. Mol. Phys. 2004, 102, 1115 $2399-2409$.

1116

(51) Hariharan, P. C.; Pople, J. A. The influence of polarization 1117 functions on molecular orbital hydrogenation energies. Theor. Chim. 1118 Acta 1973, 28, 213-222.

1119

(52) Francl, M. M.; Pietro, W. J.; Hehre, W. J.; Binkley, J. S.; 1120 Gordon, M. S.; DeFrees, D. J.; Pople, J. A. Self-consistent molecular 1121 orbital methods. XXIII. A polarization-type basis set for second-row 1122 elements. J. Chem. Phys. 1982, 77, 3654-3665.

1123

(53) Miertuš, S.; Scrocco, E.; Tomasi, J. Electrostatic interaction of a 1124 solute with a continuum. A direct utilizaion of $\mathrm{AB}$ initio molecular 1125 potentials for the prevision of solvent effects. Chem. Phys. 1981, 55, 1126 $117-129$.

1127

(54) Improta, R.; Barone, V.; Scalmani, G.; Frisch, M. J. A state- 1128 specific polarizable continuum model time dependent density 1129 functional theory method for excited state calculations in solution. 1130 J. Chem. Phys. 2006, 125, No. 054103. 
1132 (55) Frisch, M. J.; Trucks, G. W.; Schlegel, H. B.; Scuseria, G. E.; 1133 Robb, M. A.; Cheeseman, J. R.; Scalmani, G.; Barone, V.; Mennucci, 1134 B.; Petersson, G. A., et al., Gaussian 09, Revision D.01. Gaussian 09, 1135 (Revision D.01). Gaussian, Inc., Wallingford CT 2009.

1136 (56) Gomez, E.; Gutiérrez, M.; Moreno, M.; Hisaki, I.; Nakagawa, 1137 S.; Douhal, A. Spectroscopy and dynamics of dehydrobenzo[12]1138 annulene derivatives possessing peripheral carboxyphenyl groups: 1139 theory and experiment. Phys. Chem. Chem. Phys. 2018, 20, 741511407427.

1141 (57) Organero, J. A.; Tormo, L.; Douhal, A. Caging Ultrafast Proton 1142 Transfer and Twisting Motion of 1-Hydroxy-2-Acetonaphthone. 1143 Chem. Phys. Lett. 2002, 363, 409-414.

1144 (58) Randino, C.; Ziółek, M.; Gelabert, R.; Organero, J. A.; Gil, M.; 1145 Moreno, M.; Lluch, J. M.; Douhal, A. Photo-deactivation pathways of 1146 a double H-bonded photochromic Schiff base investigated by 1147 combined theoretical calculations and experimental time-resolved 1148 studies. Phys. Chem. Chem. Phys 2011, 13, 14960-14972.

1149 (59) Gil, M.; Douhal, A. Femtosecond Dynamics of a Cardiotonic 1150 Medicine (Milrinone) in Neutral Water. Chem. Phys. Lett. 2006, 428, 1151 174-177.

1152 (60) Douhal, A.; Lahmani, F.; Zewail, A. H. Proton-transfer reaction 1153 dynamics. Chem. Phys. 1996, 207, 477-498.

1154 (61) Hynes, J. T.; Klinman, J. P.; Limbach, H.-H.; Schowen, R. L. 1155 Hydrogen-Transfer Reactions; Wiley-VCH: Weinheim, Germany, 2007; 1156 Vol. 1-4.

1157 (62) Zhou, P.; Han, K. Unraveling the Detailed Mechanism of 1158 Excited-State Proton Transfer. Acc. Chem. Res. 2018, 51, 1681-1690. 1159 (63) Wirz, J. In Excited States in Organic Chemistry and Biology; 1160 Reidal Publishing Company, 1977; p 283-294.

1161 (64) Wirz, J. Electronic Structure and Photophysical Properties of 1162 Planar Conjugated Hydrocarbons with a 4n-Membered Ring; Springer: 1163 Dordrecht 1977; Vol. 10.

1164 (65) Kumpulainen, T.; Lang, B.; Rosspeintner, A.; Vauthey, E. 1165 Ultrafast Elementary Photochemical Processes of Organic Molecules 1166 in Liquid Solution. Chem. Rev. 2017, 117, 10826-10939.

1167 (66) Braem, O.; Penfold, T. J.; Cannizzo, A.; Chergui, M. A 1168 femtosecond fluorescence study of vibrational relaxation and cooling 1169 dynamics of UV dyes. Phys. Chem. Chem. Phys. 2012, 14, 3513-3519. 1170 (67) Alarcos, N.; Gutierrez, M.; Liras, M.; Sánchez, F.; Douhal, A. 1171 An abnormally slow proton transfer reaction in a simple HBO 1172 derivative due to ultrafast intramolecular-charge transfer events. Phys. 1173 Chem. Chem. Phys. 2015, 17, 16257-16269.

1174 (68) Hisaki, I.; Nakagawa, S.; Tohnai, N.; Miyata, M. A C31175 Symmetric Macrocycle-Based, Hydrogen-Bonded, Multiporous Hex1176 agonal Network as a Motif of Porous Molecular Crystals. Angew. 1177 Chem. Int. Ed. 2015, 54, 3008-3012.

1178 (69) Hisaki, I.; Ikenaka, N.; Tohnai, N.; Miyata, M. Polymorphs of 1179 layered assemblies of hydrogen-bonded hexagonal networks caused by 1180 conformational frustration. Chem. Commun. 2016, 52, 300-303.

1181 (70) Hisaki, I.; Nakagawa, S.; Sato, H.; Tohnai, N. Alignment of 1182 paired molecules of C60 within a hexagonal platform networked 1183 through hydrogen-bonds. Chem. Commun. 2016, 52, 9781-9784. 